\title{
Load-bearing behavior of spliced glass beams under bending action
}

\author{
René Ringli · Thomas Vogel
}

Received: 30 November 2015 / Accepted: 29 January 2016 / Published online: 23 February 2016

(C) Springer International Publishing Switzerland 2016
Abstract The load-bearing behavior of spliced struc- outer panes lead to higher stress concentrations in inner tural glass beams under bending action is investigated using FE simulations. Since geometric singularities occur in spliced structural glass beams, stress concen- trations arise, which are the key issue regarding the bearing capacity due to the brittle failure mode of glass. FE simulations show that stress concentration factors of more than 2.6 may occur within the cross section of spliced joints. Furthermore, the simulations show that these stress concentrations highly depend on the stiff- ness of the interlayer material, which in turn strongly depends on the temperature and the load duration. In case of low interlayer stiffness, the stress concentra- tions are low as well, but the zone of influence of a joint is large. In case of a high interlayer stiffness, the zone of influence of a joint is small and therefore, the force in the spliced pane has to be transferred in a small area leading to high stress concentrations. Consequen- tially, different load scenarios with different assump- tions regarding the stiffness of the interlayer material, have to be considered in order to get a safe design. In addition, an interaction of neighboring joints cannot be excluded and therefore, a simulation of the entire struc- ture is strongly recommended. Optimized joint designs, such as thinner outer panes or the use of an interme- diate pane, significantly reduce the stress concentra- tions in case of outer spliced joints. However, thinner
R. Ringli ( $\varangle)$
ETH Zurich, Institute for Structural Engineering (IBK),
Zurich, Switzerland
e-mail: ringli@ibk.baug.ethz.ch spliced joints, which may result in a relocation of the critical stress concentration within the beam.
Keywords Spliced structural glass beam - FE simulation $\cdot$ Stress concentration $\cdot$ Bending action

\section{List of symbols}

\begin{tabular}{|c|c|}
\hline$\alpha$ & Level of significance $(-)$ \\
\hline$b_{1}, b_{2}, b_{3}$ & $\begin{array}{l}\text { Unknown parameters of the assumed } \\
\text { regression function (-) }\end{array}$ \\
\hline$b_{\text {stat }}$ & Statically relevant width $(\mathrm{mm})$ \\
\hline$b_{\text {tot }}$ & Total beam width $(\mathrm{mm})$ \\
\hline$\beta$ & Angle of spread $\left(^{\circ}\right)$ \\
\hline E & Young's modulus $\left(\mathrm{N} / \mathrm{mm}^{2}\right)$ \\
\hline$(E I)_{F E M}$ & $\begin{array}{l}\text { Global stiffness of the beam based on the } \\
\text { FE simulation }\left(\mathrm{Nmm}^{2}\right)\end{array}$ \\
\hline$(E I)_{t h}$ & $\begin{array}{l}\text { Theoretical global stiffness of the beam } \\
\text { based on a monolithic cross section } \\
\left(\mathrm{Nmm}^{2}\right)\end{array}$ \\
\hline$F$ & Force $(\mathrm{N})$ \\
\hline$F_{a}$ & Force applied in the analytical model (N) \\
\hline$G_{\text {int }}$ & Interlayer shear stiffness $\left(\mathrm{N} / \mathrm{mm}^{2}\right)$ \\
\hline$h$ & Beam height $(\mathrm{mm})$ \\
\hline$I$ & Second moment of area $\left(\mathrm{mm}^{4}\right)$ \\
\hline$L_{\text {inter }}$ & Length of intermediate pane (mm) \\
\hline$L_{J}$ & Length of a single joint (mm) \\
\hline$L_{\text {tot }}$ & Total beam length $(\mathrm{mm})$ \\
\hline$L_{t}$ & Overall joint length (mm) \\
\hline
\end{tabular}




$\begin{array}{ll}L_{O} & \text { Overlapping length }(\mathrm{mm}) \\ L_{1} & \text { Distance between support and load } \\ & \text { introduction point (mm) } \\ \Lambda & \text { Stress concentration factor }(-) \\ M & \text { Bending moment }(\mathrm{Nmm}) \\ m & \text { Total number of joints in a beam }(-) \\ n & \text { Total number of glass layers }(-) \\ Q & \text { Concentrated force }(\mathrm{N}) \\ q & \text { Line load }(\mathrm{N} / \mathrm{mm}) \\ T & \text { Temperature }\left({ }^{\circ} \mathrm{C}\right) \\ t & \text { Number of load types in a load } \\ & \text { scenario }(-) \\ t_{i} & \text { Thickness of interlayer material }(\mathrm{mm}) \\ t_{p} & \text { Thickness of a single pane }(\mathrm{mm}) \\ v & \text { Poisson ratio }(-) \\ \sigma_{\text {max }} & \text { Maximal stress in a specific area }\left(\mathrm{N} / \mathrm{mm}^{2}\right) \\ \sigma_{g r o s s} & \text { Stress based on the gross cross } \mathrm{section} \\ & \left.\text { (N/mm }{ }^{2}\right) \\ \sigma_{1} & \text { Maximal principal tensile stress }\left(\mathrm{N} / \mathrm{mm}^{2}\right) \\ \omega & \text { Stiffness ratio }(-) \\ x, y, z & \text { Cartesian coordinates }\end{array}$

\section{Introduction}

A relatively new application of structural glass beams is spliced structural glass beams. These beams consist of several glass panes in the longitudinal, as well as, in the lateral direction. Thereby, the different glass layers are laminated together during an autoclave process, using an interlayer material with a significantly lower stiffness than the glass panes (see Sect. 2.2). The interlayer material is placed between the glass layers and also in the gap of each joint in order to balance possible unevenness and production tolerances. The concept of such a structure is schematically shown in Fig. 1. The existing joints can be grouped into two groups according to their characteristics: (i) inner spliced joints and (ii) outer spliced joints.

The advantage of such structures is the possibility to overcome the maximum length of a single glass pane. Hence, new applications are possible such as large roof beams to build an all transparent roof. The drawback of spliced structural glass beams is, however, that geometric singularities within the beam lead to stress concentrations next to each joint. These stress concentrations are the key issue regarding the bearing capacity due to the brittle failure mode of glass. Known stress concentration factors are not applicable because of the laminate structure of the glass beam and the non-homogeneous cross section (see Pilkey and Pilkey 2008).

The idea of such a structure is not new and goes back to a patent, granted in 1995 to a German firm (Bischoff 1993). Currently, spliced structural glass beams are no longer under patent protection, because the patent holder ceased to pay the annual fees several years ago. The patent protection would have been lost in 2013 at the latest, since any patent protection expires after 20 years (exceptions are possible for pharmaceuticals). In 2010, a Swiss glass manufacturer, revived the concept and exhibited a spliced structural glass beam with a total length of $21 \mathrm{~m}$ at Glasstec. Motivated by the positive recognition of the attendees, the firm conducted different laboratory tests and FE simulations in order to investigate the load-bearing behavior and the feasi-

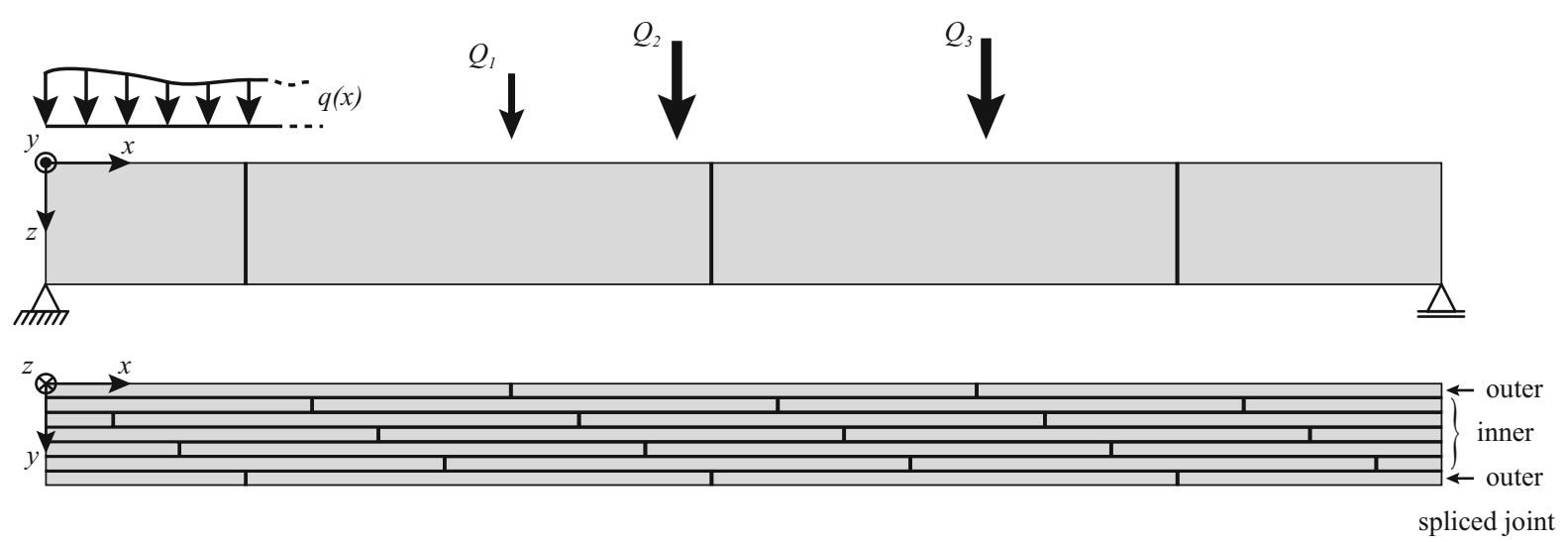

Fig. 1 Concept of a spliced structural glass beam and definition of inner and outer spliced joints; front view and top view 
bility of spliced structural glass beams (Trösch 2015). The investigations focused on the material behavior of the interlayer material, different joint configurations, long term behavior and the load-bearing behavior of pre-damaged beams. It was shown that spliced structural glass beams are feasible and the recommendation was given to use fully tempered glass in combination with SentryGlas ${ }^{\circledR}$ (SG) as interlayer material. However, an in-depth analysis of the stress flow and stress distribution within the joint cross section was not conducted and the magnitude of stress concentrations and factors affecting the stress distribution remained unknown.

In a preliminary investigation, an analytical model was developed (see Ringli and Vogel 2016) and the load-bearing behavior of a spliced five-fold laminate was investigated under pure tensile load in laboratory tests and FE simulations (see Ringli and Vogel 2015). The tests illustrated that the load-bearing behavior of a spliced laminate under tensile load depends primarily on a combination of three effects (more details are given in Sect. 2.4):

- A load redistribution according to the different stiffness of glass and interlayer material

- Bending of a sandwich element due to the lateral bending action induced by the eccentricity of the residual force

- Influence of local effects, which correspond to stress concentrations in the proper sense

This behavior may lead to stresses that are $300 \%$ larger than the ones assuming a uniformly distributed stress in the gross cross section for the analyzed configurations. Furthermore, the laboratory tests showed that the load-bearing behavior highly depends on temperature, since the stiffness of the interlayer material dramatically decreases between room temperature and $50{ }^{\circ} \mathrm{C}$. Hence, these findings have to be adapted and verified for beams under bending action.

\section{Fundamentals}

The following fundamental assumptions are made:

- Laboratory tests by Trösch (2015) showed that the failure of structural glass beams starts as glass failure and not as a cohesion or adhesion failure of the interlayer material between two glass layers.
- The maximum thickness of the entire beam $b_{t o t}$ is limited to roughly $100 \mathrm{~mm}$, because SG requires a minimal cooling rate of about $2.2^{\circ} \mathrm{C} / \mathrm{min}$ after the lamination process in the autoclave; otherwise opaque crystal structures would develop in SG (see Trösch 2015).

- Lateral stability issues are excluded in this study, because it is assumed that in practical cases the beam will be stabilized at the upper edge through the façade structure.

The basis of the investigations presented here is a four point bending test. In order to compare the results of different configurations, all loads (also dead load) are applied as concentrated forces at the two load introduction points in order to get a uniform bending action in between. Furthermore, joints between the load introduction points and the supports are avoided in order to keep the comparability of different configurations. As a consequence, the configurations investigated here should be seen as fictitious examples to gain a better understanding of the issues arising with spliced glass beams rather than examples for practical applications. Since thousands of different configurations of spliced structural glass beams are possible, the specific factors presented here are not suited to be taken one to one to practice. However, the findings regarding the fundamental load-bearing behavior and the stress flow within the beam are applicable in general.

The definitions for layers, joints and different lengths are given in Fig. 2.

The joints are named in alphabetical order and the total $n$ glass layers are named $\mathrm{S} 1$ to $\mathrm{S} n$. Furthermore, the joint length $L_{J}$, the overlap length $L_{O}$, the distance between support and load introduction point $L_{1}$ and the overall joint length $L_{t}$ are introduced.

\subsection{Joint layout}

The single glass panes can be arranged in different ways to form a spliced structural glass beam. Two selected layouts $\mathrm{K} 1$ and $\mathrm{K} 2$ are schematically shown for a seven-, eight- and nine-fold laminate (called L7, L8 and L9, respectively) in Fig. 3.

In layout K1, the panes are arranged in such a way that the distance between two neighboring joints in two adjacent panes is maximized. This condition leads to a layout where the next but one layer is spliced in the next joint. For example, in case of layout K1 for a seven- 
Fig. 2 Definition of used parameters

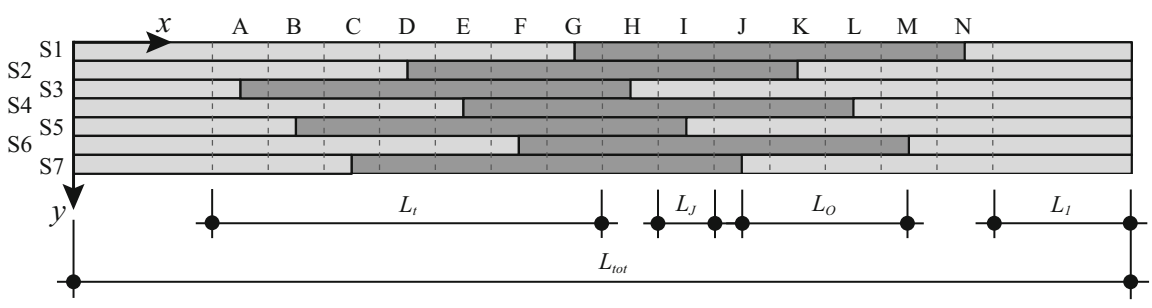

K1

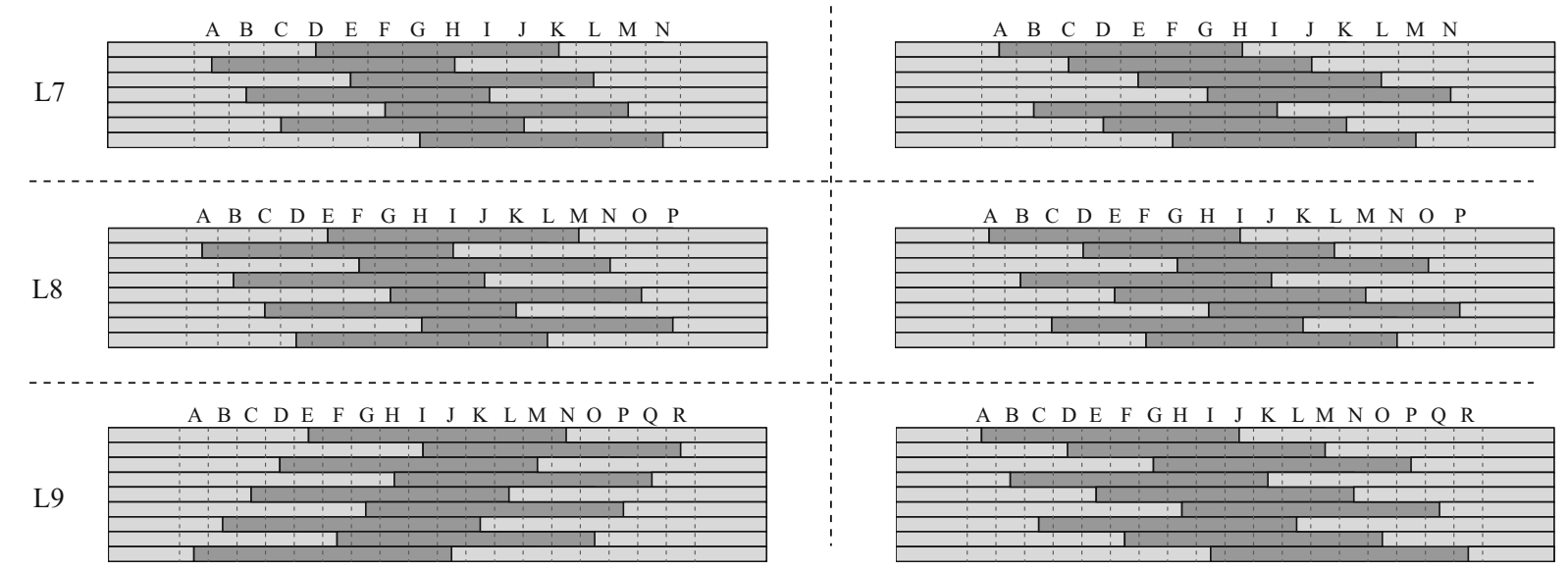

Fig. 3 Different joint layouts for seven-, eight- and nine-fold laminates; layout K1 with maximized $L_{O}$ and layout K2 with a more evenly distributed $L_{O}$ across the entire beam

fold laminate, $\mathrm{S} 1$ is spliced at joint $\mathrm{D}$ and $\mathrm{S} 3$ in the neighboring joint $\mathrm{E}$.

In layout $\mathrm{K} 2$, the focus lies not on maximizing $L_{O}$ in each layer but to come up with a configuration where $L_{O}$ is more evenly distributed across the entire beam. This results in a layout with less interlocked panes than K1.

\subsection{Material parameter}

Different environmental conditions apply during the lifetime of a spliced structural glass beam, which have a significant influence on the load-bearing behavior and especially on the stress concentrations in the joints. This is due to the fact that the stiffness of the interlayer material highly depends on temperature and load duration as illustrated in Fig. 4.

Further experimental test results presented in Trösch (2015) are in good agreement with the values provided by the producer. However, other experimental tests conducted by Callewaert (2012) indicate that the provided values are too high and the actual values are significantly lower.

A simplification of the material behavior of $\mathrm{SG}$ is to assume that it behaves linear viscoelastically. According to Boltzmann (1874), linear viscoelastic material behavior can be approximated by superposition of linear elastic material behavior considering the appropriate Young's modulus. This approach is widely used within the glass industry (Schuler 2003; Wellershoff 2007; DIBt 2011) because it is simple to use and the needed linear elastic parameters are available. The approximation of the load-bearing behavior of SG by superposition of linear elastic material behavior is justified due to the typically small deflections expected in the application of spliced structural glass beams.

If nonlinear material behavior is assumed, a superposition of results would be invalid and the entire service life must be simulated. Since reliable material properties for such a lifetime simulation are missing, trustworthy simulations are difficult to conduct. In addition, the plausibility of the obtained results is more difficult to verify when complex material models are used. 
(a)

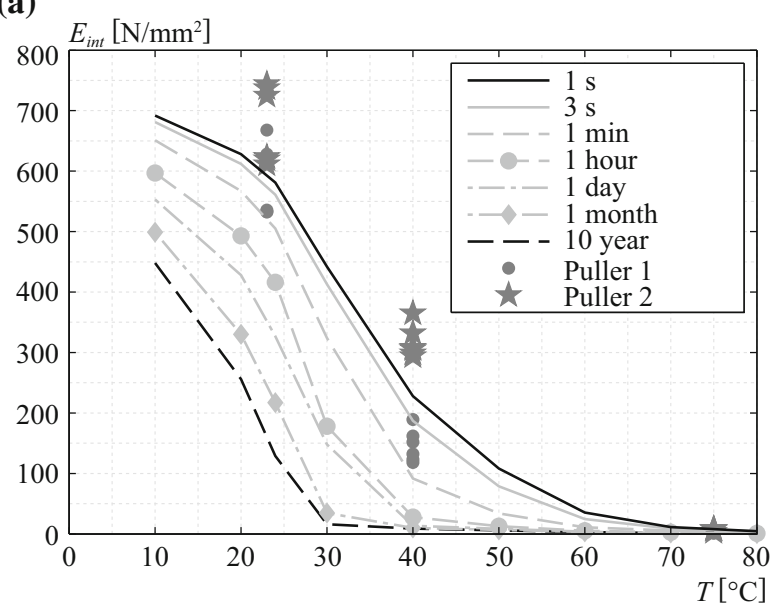

Fig. 4 Material parameter for SG for different temperatures and load durations: a Young's modulus, Puller 1 (velocity = $1 \mathrm{~mm} / \mathrm{min}$ ), Puller 2 (velocity $=100 \mathrm{~mm} / \mathrm{min}$ ); b Shear mod-

\subsection{Equations and definitions}

Previous research showed that each pane in laminated glass beams, consisting of one pane in the longitudinal direction, acts according to the beam theory (see Hess 2000; Belis et al. 2009). In consequence, the interlayer material can be neglected for the determination of the theoretical stresses based on the gross cross section $\sigma_{\text {gross }}$. Hence, $\sigma_{\text {gross }}$ arises at the lower edge of the beam due to the bending moment $M$, and can be calculated according to Eq. (1)

$\sigma_{\text {gross }}=\frac{M}{I} \cdot \frac{h}{2}=\frac{6 M}{b_{\text {stat }} h^{2}}$

where $I$ is the second moment of area of the beam section, $h$ is the beam height and $b_{\text {stat }}$ is the statically relevant width of the beam, which is equal to the sum of the thickness of all glass layers. In general, $M$ is not constant over the length of the beam and therefore, $\sigma_{\text {gross }}$ has to be calculated separately for all cross sections, which will be taken into account during design.

The stress concentration factor $\Lambda$ for each joint $i$ of the total $m$ joints within the beam is defined by Eq. (2).

$$
\Lambda_{i}=\frac{\sigma_{\max , i}}{\sigma_{\text {gross }, i}}
$$

(b)

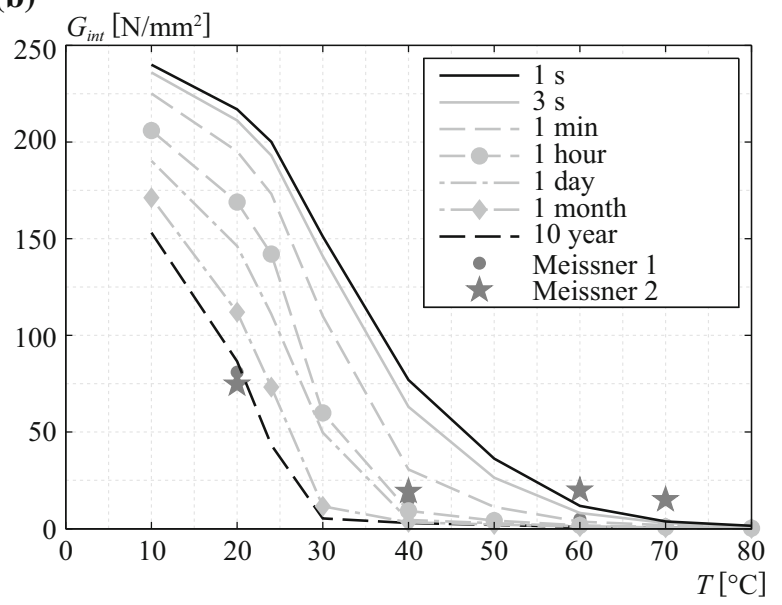

ulus, Meissner 1 (velocity $=0.5 \mathrm{~mm} / \mathrm{min}$ ), Meissner 2 (velocity $=5 \mathrm{~mm} / \mathrm{min}$ ); data source: Kuraray (2014), Puller (2012), Meissner et al. (2005)

In case of different load scenarios and stiffness assumptions regarding the material behavior of SG, the total stress concentration factor $\Lambda_{\text {tot }}$ for the specific scenario can be calculated according to Eq. (3).

$$
\begin{aligned}
\Lambda_{\text {tot }, i} & =\frac{\sum_{j=1}^{t} \Lambda_{i}\left(G_{j}\right) \cdot \sigma_{\text {gross }, i, j}}{\sum_{j=1}^{t} \sigma_{\text {gross }, i, j}} \\
& =\frac{\sum_{j=1}^{t} \Lambda_{i}\left(G_{j}\right) \cdot \sigma_{\text {gross }, i, j}}{\sigma_{\text {gross }, \text { tot }, i}}
\end{aligned}
$$

where $t$ denotes the number of different load and stiffness assumptions within one load scenario.

Subsequently, the maximal tensile stress $\sigma_{\max }$ can be calculated with Eq. (4).

$$
\begin{array}{r}
\sigma_{\text {max }}=\max \left(\Lambda_{\text {tot }, 1} \cdot \sigma_{\text {gross }, \text { tot }, 1}, \ldots,\right. \\
\left.\Lambda_{\text {tot }, m} \cdot \sigma_{\text {gross }, \text { tot }, m}\right)
\end{array}
$$

Since linear elastic material behavior is assumed and nonlinear geometric effects are neglected, the stress concentration factor is load-independent.

\subsection{Analytical model}

An analytical model was introduced in Ringli and Vogel (2016), which estimates the maximum tensile stress in the joint cross section. The model focuses on one single joint and approximates the lower edge of a beam under constant bending action as a region of pure tensile action. Based on these simplifications, the model 
estimates the stresses in the joint cross section as a superposition of three effects:

- Load redistribution due to the different stiffness of the glass and the interlayer material is calculated using an adapted slip equation. Any bending action due to eccentricity is neglected at this point. Hence, it is assumed that the tensile stresses in each glass pane are uniformly distributed.

- The residual force shows an eccentricity as a result of the new stress distribution that leads to a lateral bending action. This bending action is considered by applying a specific load case that results in a similar bending moment to an equivalent beam. The equivalent beam is then analyzed using sandwich theory.

- In the first part it was assumed that the transferred force spread equally over each pane, which results in a uniform stress distribution per pane. This assumption is not valid in the joint cross section for the adjacent pane because the transferred force does not have the possibility to spread since the force is transferred before and retransferred right after the joint. This local effect is considered by an additional local bending action. The bending moment is assumed to be the product of to the force which is transferred within a certain area before and after the joint and a lever arm of around one third of the pane thickness.

\section{Finite element model}

The FE model is a three-dimensional model implemented in the commercial software ABAQUS. Thereby, the glass panes, as well as the interlayer parts, were modelled using three dimensional second order elements. The different parts were coupled together at the contact surfaces by means of the built-in coupling restrictions (tie constraints). Furthermore, it was assumed that $\mathrm{SG}$ is not able to transfer tensile stresses in the joint, because previous research showed that the joints may open during loading and adhesive failure in the gap occurs (see Trösch 2015). As a consequence, the interlayer material in the gap was separated on half the height into two areas. Within the lower part, a low Young's modulus of $E=0.1 \mathrm{~N} / \mathrm{mm}^{2}$ was assigned and within the upper part, the same Young's modulus was assigned as in the other SG areas. This modeling technique was chosen because the built-in no tension
Table 1 Used element types and linear elastic material parameters

\begin{tabular}{llll}
\hline Material & $E\left(\mathrm{~N} / \mathrm{mm}^{2}\right)$ & $v(-)$ & Element type \\
\hline Glass & 70000 & 0.23 & C3D20R \\
SG & Var. & 0.49 & C3D20RH \\
SG joint lower part & 0.1 & 0.49 & C3D20RH \\
\hline
\end{tabular}

material characteristic, provided by $A B A Q U S$, leads to convergence problems.

All material properties were set to linear elastic and the used material parameters as well as the assigned element types are listed in Table 1.

Preliminary calculations showed that the element size does not have a significant influence on the stress distribution in the joint cross section. In order to keep the computation time in an acceptable range, four elements were used in the transversal direction ( $y$-direction) in each glass pane and three elements within each interlayer. Eight elements were used over the height. A different number of elements was used over each joint length in the longitudinal direction as illustrated in Fig. 5.

The boundary conditions were defined according to the static model as shown in Fig. 5 and the beam was linearly supported at the upper edge in order to avoid lateral buckling.

Two sets of FE simulations were performed. In the first set, configurations of different spliced structural glass beams with identical pane thickness $t_{p}$ were conducted. In the second set, configurations with optimized joint designs were performed.

Due to the characteristic material behavior of SG (see Sect. 2.2), each simulation was conducted for the following $G_{\text {int }}$ values: 2, 4, 10, 25, 50, 100, 150, 200 and $250 \mathrm{~N} / \mathrm{mm}^{2}$. The thickness of the interlayer $t_{i}$ is $1.52 \mathrm{~mm}$ for all configurations. For each configuration the forces were chosen in such a way that $\sigma_{\text {gross }}=$ $30 \mathrm{~N} / \mathrm{mm}^{2}$.

\section{FE simulations results}

All calculated stresses are shown as maximal principal tensile stresses based on $35 \%$ values. A value of $35 \%$ means that at each finite element node, shared between adjoining elements, the extrapolated stresses from the different finite elements are averaged when 


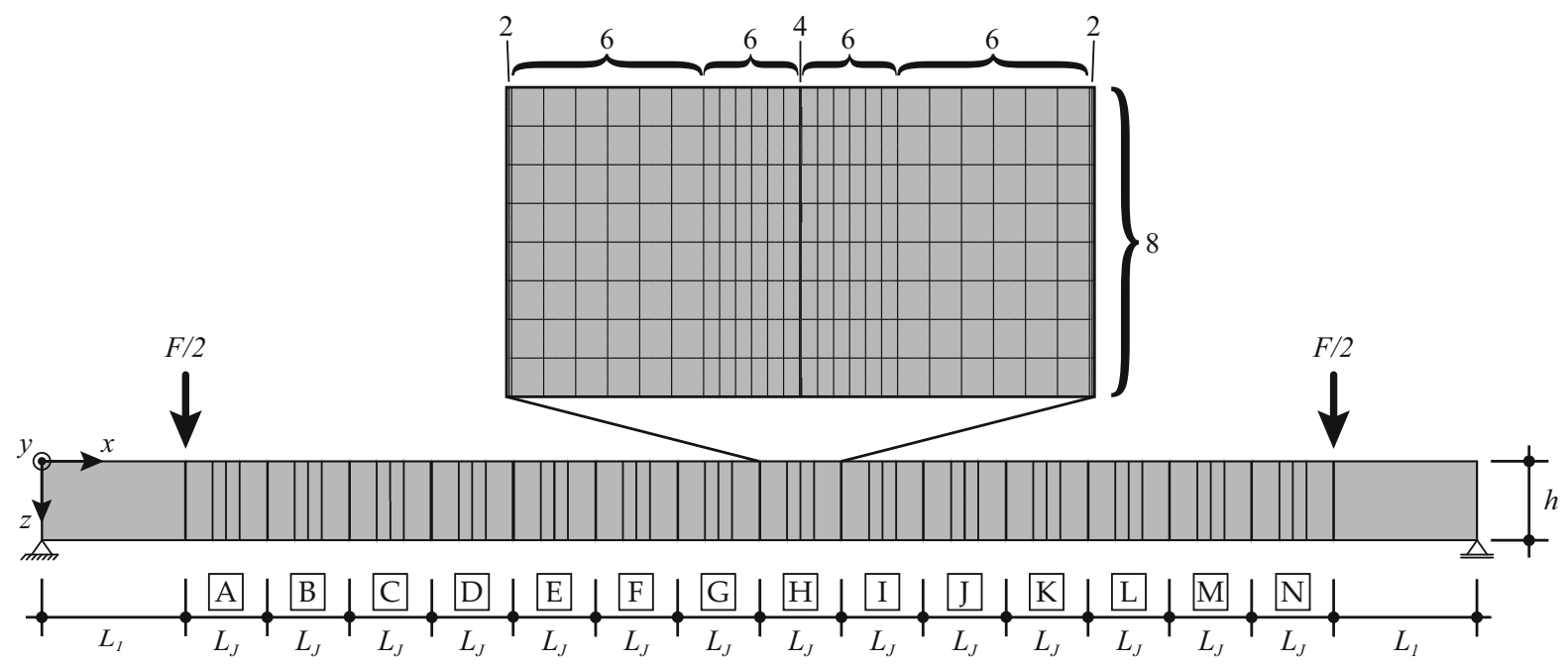

Fig. 5 Number of elements in the different element areas of the FE model and static model of the four point bending test using the example of a seven-fold laminate

they do not differ by more than $35 \%$. A value of $35 \%$ is chosen because this is considered to be a good compromise between getting a smoothing effect and not over smoothing the raw data.

All simulated configurations from the first set are listed in Table 2. Simulations were made for seven-, eight-, and nine-fold laminates with $L_{\text {tot }}=10,16$ and $20 \mathrm{~m}$ and $h=700,900$ and $1100 \mathrm{~mm}$, respectively.

\subsection{Sevenfold laminate}

In a first step, the stress distribution for a low and a high interlayer stiffness for the configuration L7_12_K1_ 16_h900 is discussed on a general level. In a second step, the stress concentration factors depending on the interlayer stiffness are shown and compared. Thereby, the nonlinear regression function $y(x)$ defined by Eq. (5) is used to illustrate the nonlinear dependence on $G_{\text {int }}$.

$y(x)=b_{1} \cdot\left(1-\mathrm{e}^{-b_{2} \cdot x}\right)+b_{3}$

The unknown parameters $b_{1}, b_{2}$ and $b_{3}$ were estimated with the least-square estimation method according to Levenberg-Marquard (see Seber and Wild 2003). The level of significance for the confidence level was set to $\alpha=0.05$.

The first and the last joint were not considered in the analysis in order to avoid the influence of the supports and keep the results comparable between the different configurations.

\subsubsection{Load-bearing behavior in general: using example configuration L7_12_K1_16_h900}

The stresses along the $x$-axis, which arise at the lower edge, are illustrated in Fig. 6a for each pane axis assuming $G_{i n t}=200 \mathrm{~N} / \mathrm{mm}^{2}$. The stress distributions along $y$ for the joints B to $\mathrm{H}$ are illustrated in Fig. $6 \mathrm{~b}$.

The stress distributions in the longitudinal direction show that the stresses at joints in the spliced layer deand increase within a narrow area around each joint and rapidly normalize back to the gross level of $30 \mathrm{~N} / \mathrm{mm}^{2}$. Hence, the stresses are more or less on the level of $\sigma_{\text {gross }}$ in the bigger part of the beam. It can, therefore, be concluded that the joints do not influence each other and the joint layout does not affect the overall loadbearing behavior.

Considering the stress distribution along the $y$-axis, it can be seen that $\sigma_{\max }$, which is approximately 2.5 times larger than $\sigma_{\text {gross }}$, occurs directly next to the spliced pane in the outer spliced joints D and G. The stress distribution in these joints shows the characteristics, as expected from the previous investigations (Ringli and Vogel 2015, 2016), as a combination of load redistribution due to the different stiffness, lateral bending of a sandwich element and local effects. 


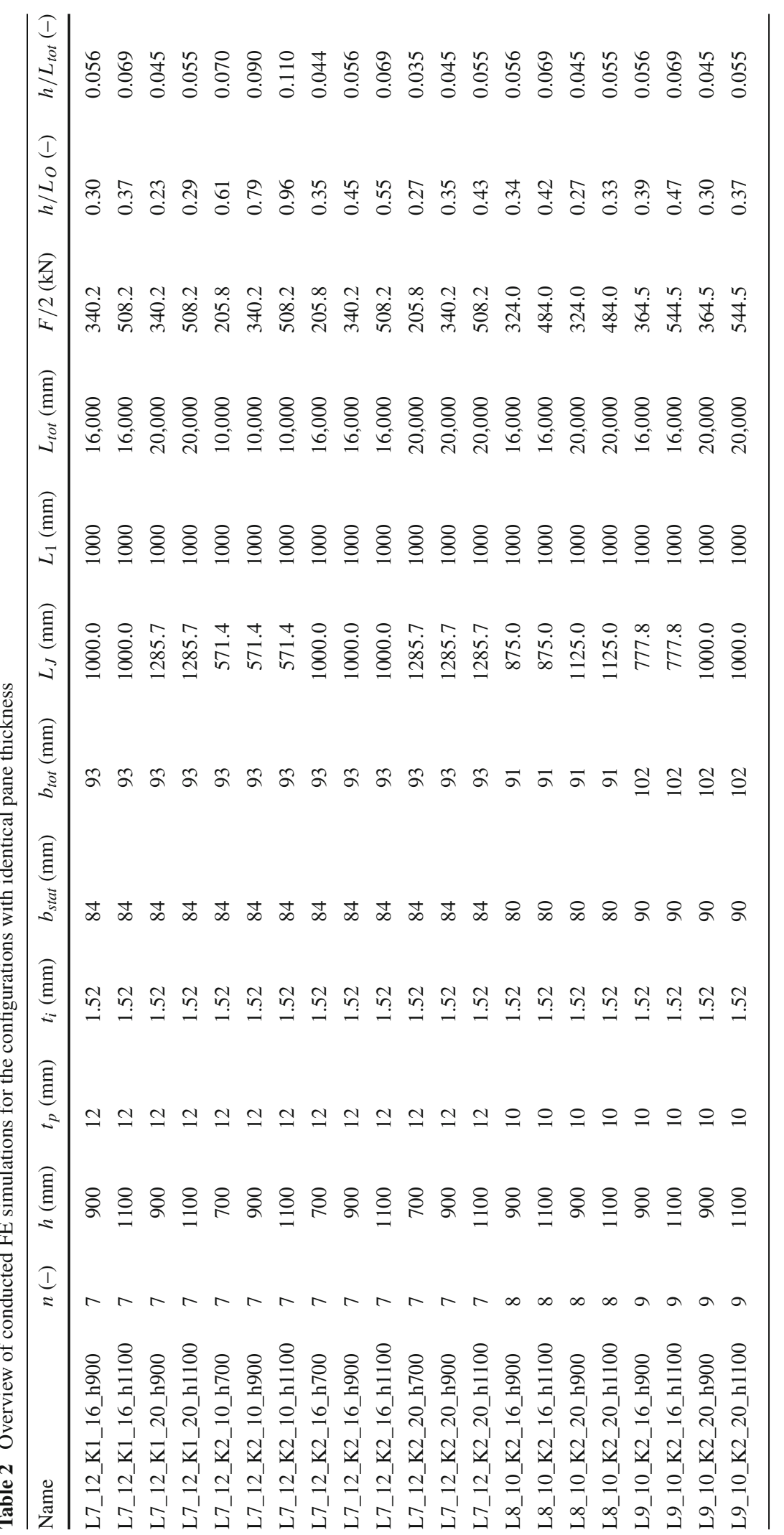


(a)

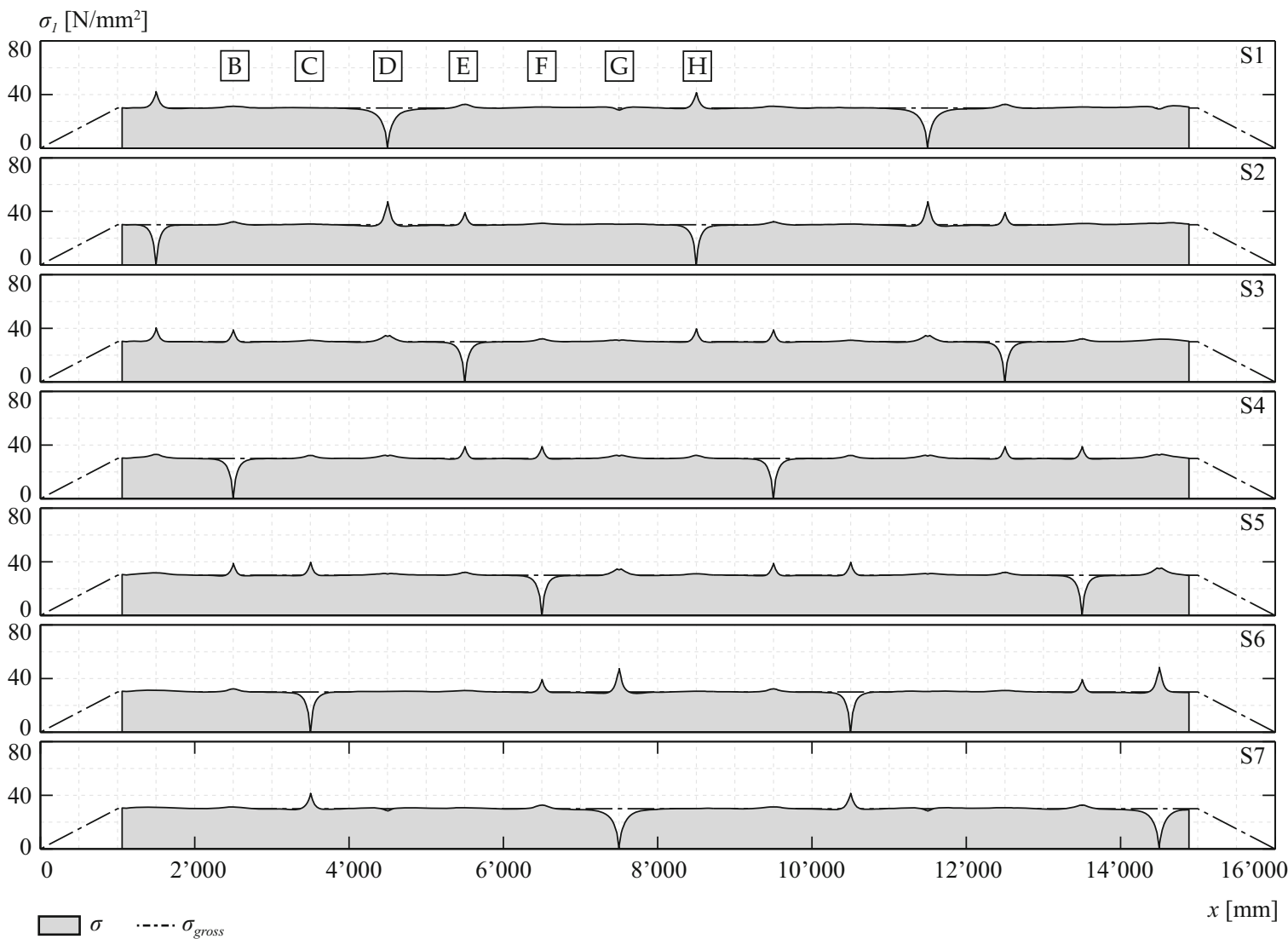

(b)

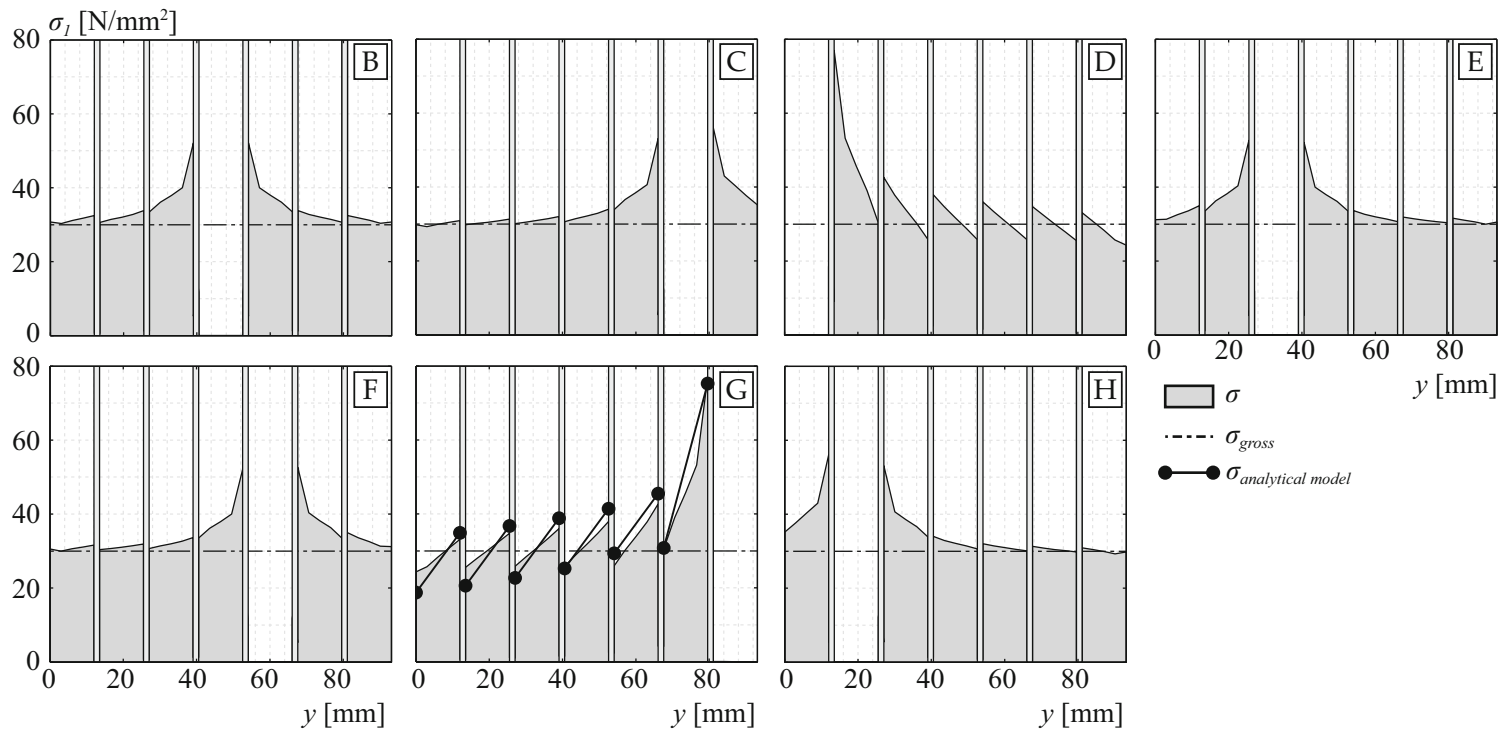

Fig. 6 Maximal principal tensile stress on the lower beam edge for configuration L7_12_K1_16_h900 assuming $G_{\text {int }}=200$ N/mm ${ }^{2}$ : a stresses along $x$ at each pane axis; $\mathbf{b}$ stresses along $y$ in the joint cross section for the joints $\mathrm{B}$ to $\mathrm{H}$ 
In addition, the stress distribution in joint $\mathrm{G}$ is compared to the results obtained from the analytical model. Thereby, the following parameters were used as input variables for the analytical model: angle of spread $\beta=15^{\circ}$, linear increasing load for $G_{i n t} \geq 25 \mathrm{~N} / \mathrm{mm}^{2}$, concentrated force for $G_{i n t}<25 \mathrm{~N} / \mathrm{mm}^{2}$ and $L=L_{J}$. The force $F_{a}$, applied in the analytical model, can be calculated according to Eq. (6).

$F_{a}=\sigma_{\text {gross }} \cdot b_{\text {stat }}=\frac{6 M}{b_{\text {stat }} \cdot h^{2}} \cdot b_{\text {stat }}=\frac{6 M}{h^{2}}$

The comparison shows that the stresses obtained from the analytical model are in good agreement with the stresses obtained from the FE simulations. Consequently, it can be concluded that the analytical model is appropriate to gain a better understanding of the loadbearing behavior of spliced structural glass beams and to verify the plausibility of the results obtained in FE simulations.

An inner spliced joint leads to significantly lower $\sigma_{\text {max }}$ in comparison to an outer spliced joint. The largest increase occurs in the outer continuous pane in the joints where S2 or S6 is spliced. Again, the panes adjacent to the spliced pane are most affected by the joint.

In case of a low $G_{i n t}=2 \mathrm{~N} / \mathrm{mm}^{2}$, the load-bearing behavior changes significantly. In Fig. 7a the stresses at each pane axis along $x$ are plotted and in Fig. 7b the stress distribution in $y$ direction for the joints $\mathrm{B}$ to $\mathrm{H}$.

The stress in the spliced pane de- and increases more gradually compared to the change in case of a high $G_{\text {int }}$. In consequence, the stresses do not normalize on the level of $\sigma_{\text {gross }}$ between neighboring joints. The zone of influence for each joint increases and influences the stress distribution of the neighboring joints. Considering joint $\mathrm{C}$, the stresses in $\mathrm{S} 1$ and $\mathrm{S} 4$ are lower than expected if no interaction occurs between the joints. The stresses in S1 and S4 are lower because S1 and $\mathrm{S} 4$ are spliced in the neighboring joints $\mathrm{B}$ and $\mathrm{D}$. As a result, an individual analysis of each joint is not advisable and the beam as a whole has to be analyzed.

Since the zone of influence increases, the force is transferred over a larger area around the joint. This leads to a lower $\sigma_{\max }$ because the transferred force is spread more equally, reducing local effects. Hence, $\sigma_{\max }$ decreases from $2.6 \cdot \sigma_{\text {gross }}$ in case of a high $G_{\text {int }}$ to $1.75 \cdot \sigma_{\text {gross }}$ in case of a low interlayer stiffness.

Results from the analytical model deviate from the finite element analyses due to the fact that only one single joint is considered in the analytical model that does not account for interaction between neighboring joints.
Theoretically, it is possible to consider the interaction between joints in the analytical model through an adaptation of the boundary conditions. In practice, however, this is not feasible since the system spliced structural glass beam is multiple statically indeterminate, which would require an analysis of the whole system.

Although the maximal stress concentration in an outer spliced joint is considerably lower in comparison to the obtained stress concentrations for high $G_{i n t}$, the maximal stress concentration still occurs at outer spliced joints. For cases with a low interlayer stiffness, the difference in $\Lambda$ between inner and outer spliced joints is less significant.

\subsubsection{Influence of joint layout}

In order to compare the different joint layouts $\mathrm{K} 1$ and $\mathrm{K} 2$, the calculated $\Lambda$ values are illustrated in Fig. 8 for the different configurations with a beam height of $900 \mathrm{~mm}$. Figure 8 shows the $\Lambda$ values obtained for cases with inner spliced joints in (a) and for an outer spliced joint in (b).

The regression function stops at $G_{i n t}=2 \mathrm{~N} / \mathrm{mm}^{2}$. An extrapolation to lower $G_{i n t}$ and even $G_{i n t}=0$ is not tolerable as spliced structural glass beams need a stiffness of the interlayer material greater than 0 in order to ensure the force transfer between the different glass panes.

Inner spliced joint ( 22 or S6 spliced) In case of an inner spliced joint, the increase of $\Lambda$ from 1.6 to 1.9 is rather small. The different joint layouts have a small influence on the obtained stresses. In case of low $G_{\text {int }}$, the configurations $\mathrm{K} 1$ show a slightly higher $\Lambda$ because the beam is more interlocked compared to the $\mathrm{K} 2$ configuration. Through the interlocking and the consequential stiffer behavior, the continuous panes attract force, which leads to higher stresses and strains.

Outer spliced joint The obtained $\Lambda$ highly depend on $G_{i n t}$ and ranges from 1.7 for low $G_{i n t}$ to 2.6 for a high interlayer stiffness. Initially, the gradient of the increase of $\Lambda$ is high and becomes more gradual thereafter. Again, there are only marginal differences between the configurations $\mathrm{K} 1$ and $\mathrm{K} 2$.

Both values for inner spliced joints as well as the values for outer spliced joints show a good agreement with the regression function and the adjusted coefficient of determination for each configuration is greater than 0.98 . 
(a)

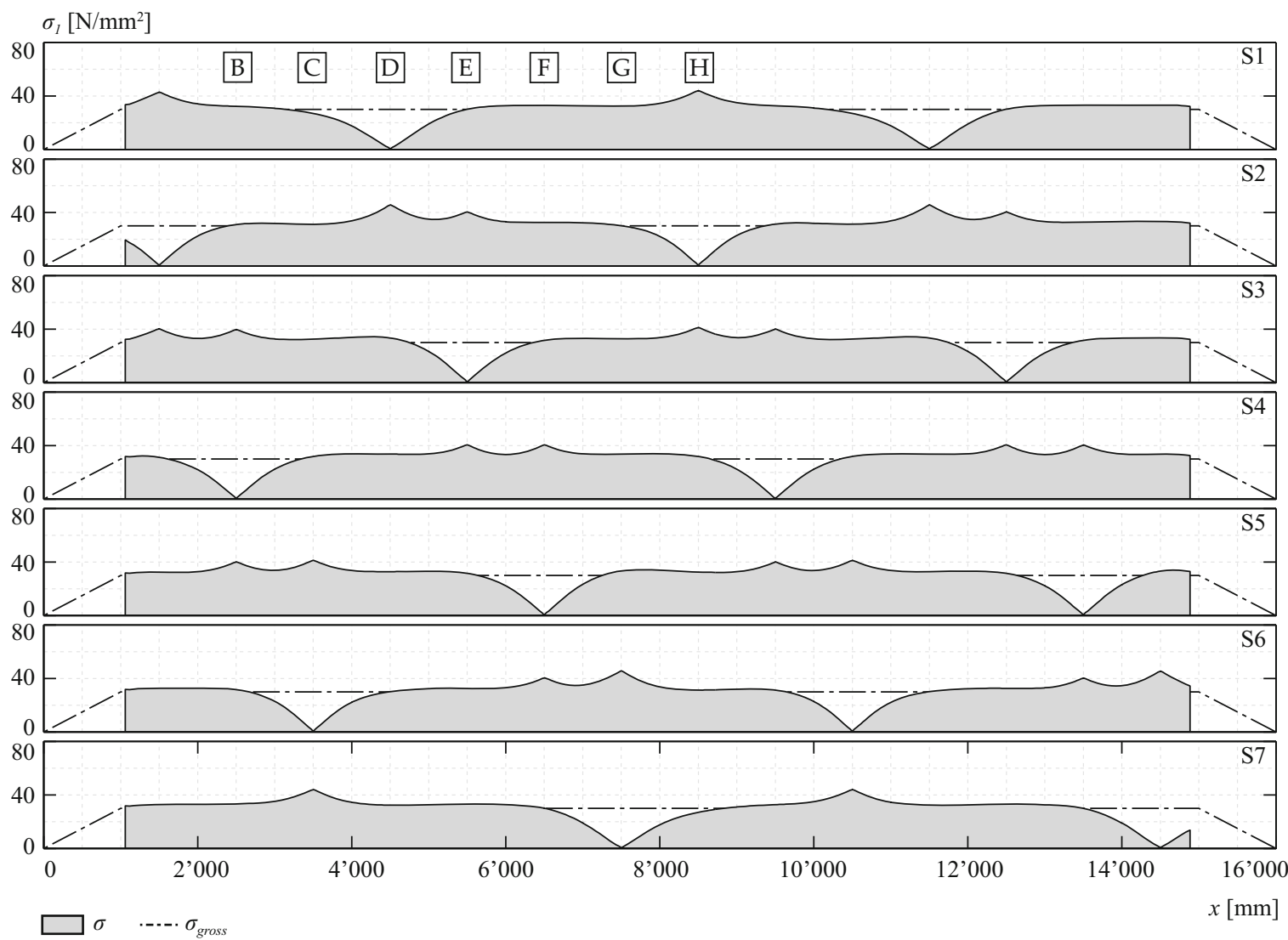

(b)

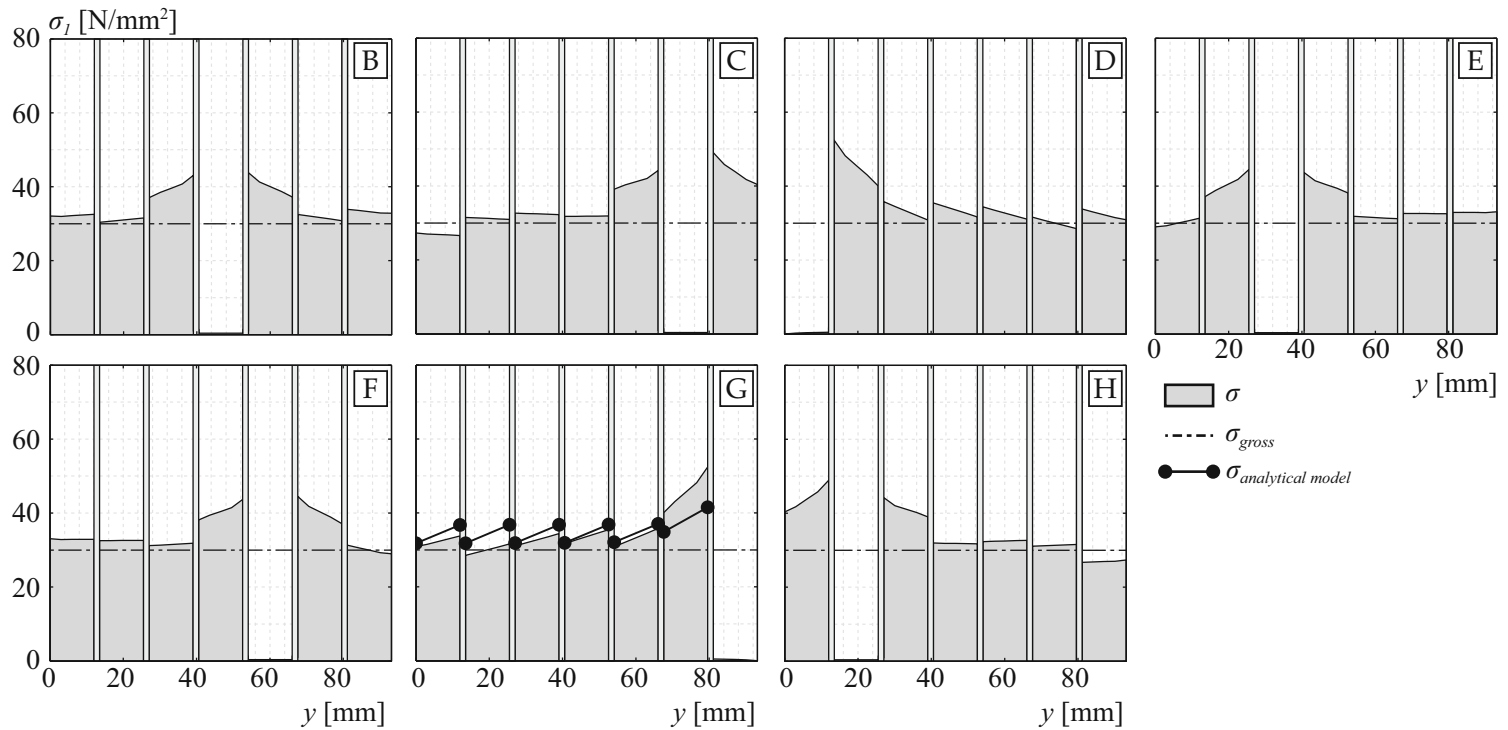

Fig. 7 Maximal principal tensile stress on the lower beam edge for configuration L7_12_K1_16_h900 assuming $G_{\text {int }}=2$ N/mm ${ }^{2}$ : a stresses along $x$ at each pane axis; $\mathbf{b}$ stresses along $y$ in the joint cross section for the joints $\mathrm{B}$ to $\mathrm{H}$ 
(a)

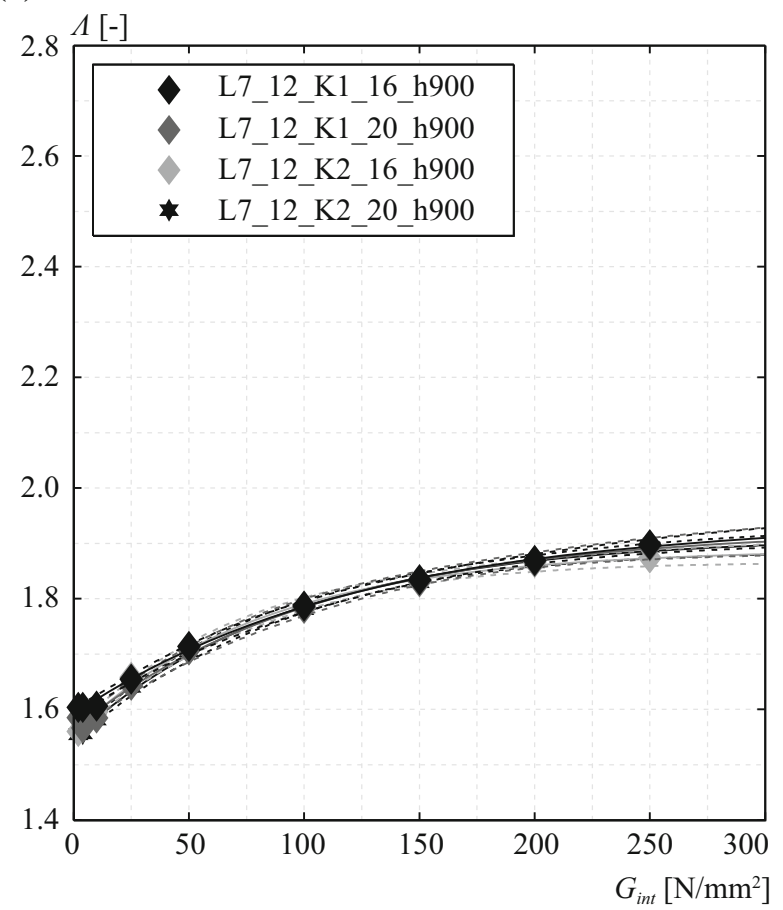

Fig. 8 Stress concentration factor $\Lambda$ variation with $G_{i n t}$ for the configurations L7_12_K1_16_h900, L7_12_K1_20_h900, L7_12_K2_16_h900 and L7_12_K2_20_h900 with the esti-

The overall performance of configuration $\mathrm{K} 2$ is better due to the fact that the individual panes are less interlocked, which leads in case of a low $G_{\text {int }}$ to lower $\Lambda$ values. As a result, further investigations will focus on beams with layout $\mathrm{K} 2$.

The goodness of fit of the proposed regression function in Eq. (5) is very high for the inner spliced joints (see Fig. 8a) but somewhat limited for outer spliced joints (see Fig. 8b). Hence, another function for outer spliced joints could improve the goodness of fit. However, to avoid an additional function, and since the fit is reasonably good, the function proposed in Eq. (5) is used further on.

\subsubsection{Influence of beam length and height}

The obtained $\Lambda$ for different beams with varying lengths and heights are illustrated in Fig. 9 for the configurations L7_12_K2_....

Inner spliced joint (S2 or S6 spliced) In case of an inner spliced joint, there is no significant difference (b)

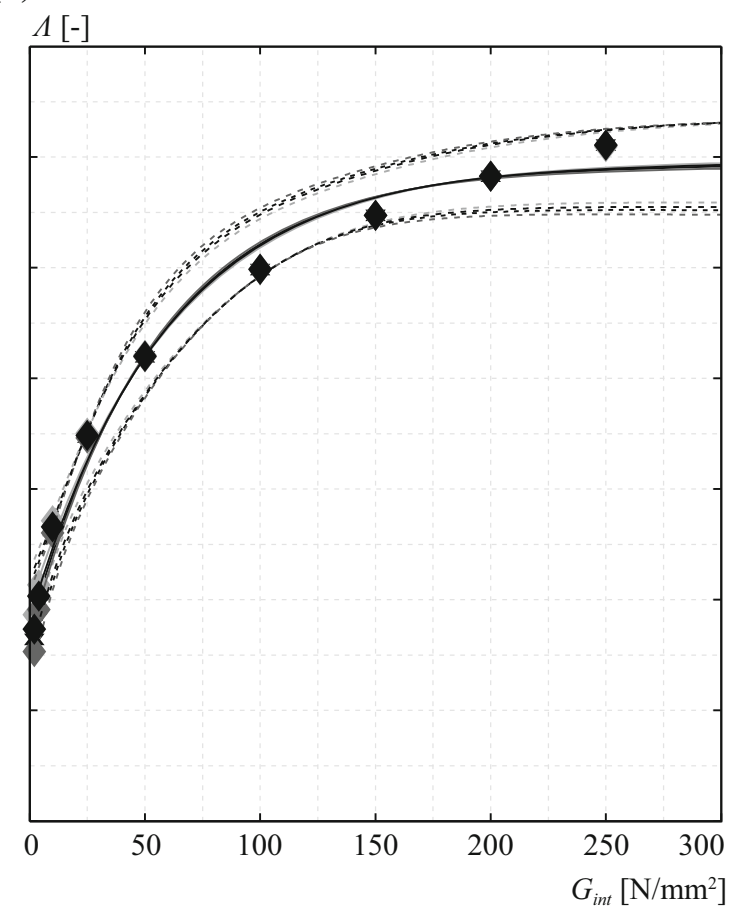

mated nonlinear regression function: a inner spliced joint (S2 or S6 spliced); b outer spliced joint

between the different configurations. The obtained $\Lambda$ are all in the range from 1.6 to 1.9 .

Outer spliced joint In case of an outer spliced joint, $h$ has an influence on the stress concentrations. An increase of the beam height leads to higher $\Lambda$. This is because the overall stiffness of the beam decreases in comparison to a monolithic beam (see next Sect. 4.1.4), which leads to larger deflections and, in turn, results in higher stresses in the spliced beam.

\subsubsection{Global stiffness}

The global bending stiffness of a spliced structural glass beams can be approximated according to the principle of virtual work, which results in Eq. (7). Equation (7) is based on the mid span displacement $w$ obtained from the FE simulations and $F, L_{1}$ and $L_{\text {tot }}$ according to table 2. The last part of Eq. (7) accounts for the displacements, which are a consequence of the shear force along $L_{1}$ and takes into account the cross section area, $I$ and the Poisson ration $v$. 
(a)

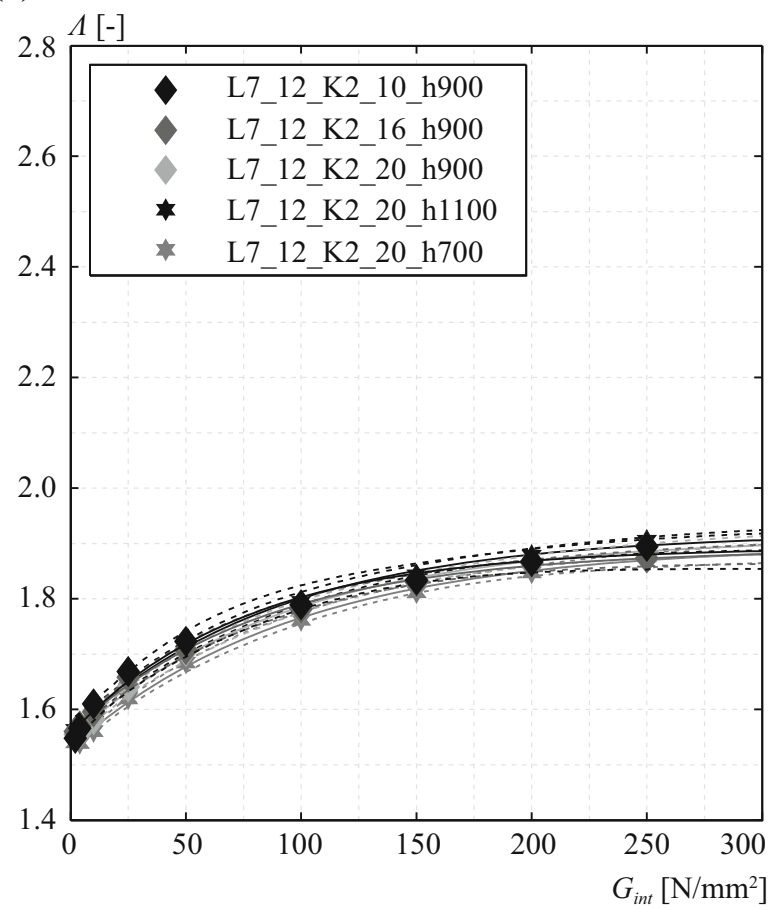

Fig. 9 Stress concentration factor $\Lambda$ variation with $G_{\text {int }}$ for the configurations L7_12_K2_10_h900, L7_12_K2_16_h900, L7_12_K2_20_h900, L7_12_K2_20_h1100 and L7_12_K2_

$$
\begin{aligned}
(E I)_{F E M}= & \frac{F L_{1}^{3}}{6 w}+\frac{F L_{1}^{2}\left(L_{t o t}-2 L_{1}\right)}{4 w} \\
& +\frac{F L_{1}\left(\frac{L_{t o t}}{4}-\frac{L_{1}}{2}\right)\left(L_{t o t}-2 L_{1}\right)}{4 w} \\
& +\frac{6 F L_{1}(1+v) I}{5 w b_{\text {stat }} h}
\end{aligned}
$$

The fundamental assumption is that the stiffness is constant over the entire length of the beam and no additional shear deformations occur, which is only approximately true in practice. Hence, the bending stiffness calculated according to Eq. (7) corresponds to a fictitious smeared bending stiffness.

The theoretical stiffness of a monolithic beam is calculated with Eq. (8)

$$
(E I)_{t h}=E \cdot \frac{b_{s t a t} \cdot h^{3}}{12}
$$

with $E=70,000 \mathrm{~N} / \mathrm{mm}^{2}$ and $h$ and $b_{\text {stat }}$ according to Table 2. (b)

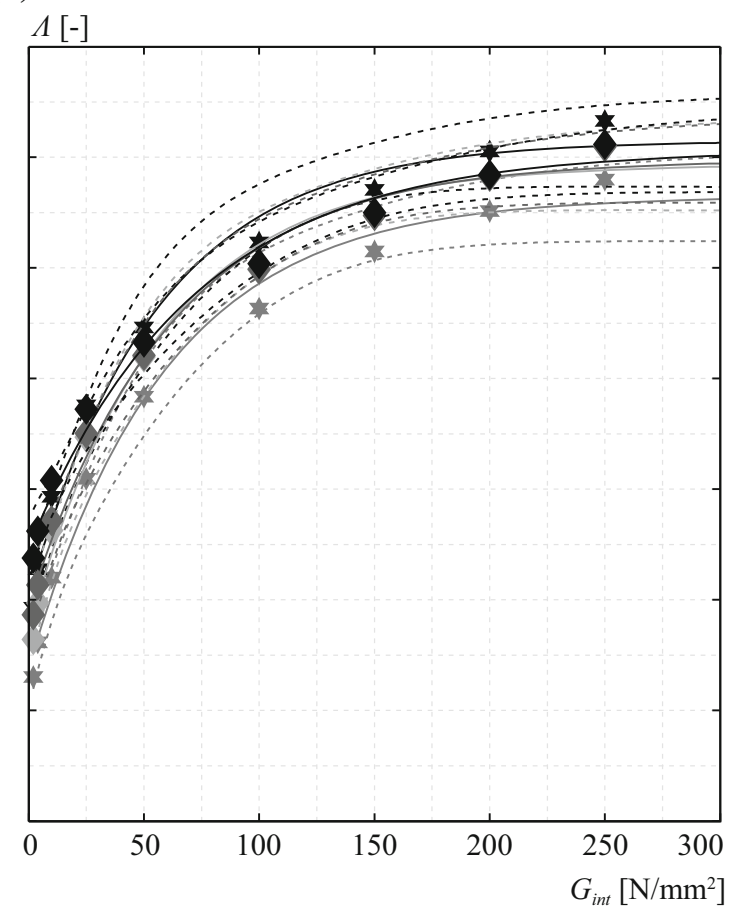

20_h700 with the estimated nonlinear regression function: a inner spliced joint (S2 or S6 spliced); b outer spliced joint

The stiffness ratio $\omega$ is defined by Eq. (9).

$\omega=\frac{(E I)_{F E M}}{(E I)_{t h}}$

Applying Eq. (9) on several configurations and different $G_{i n t}$, the stiffness ratio in dependence of beam slenderness shows the trend illustrated in Fig. 10.

Figure 10 shows indirect correlation between $\omega$ and slenderness. This effect is more distinct in cases with low $G_{i n t}$ than in cases with high $G_{i n t}$. This behavior can be explained by the fact that increasing beam height $h$ and constant $L_{\text {tot }}$ activates a larger area of the interlayer material, which has a significantly lower stiffness, leading to a lower global stiffness compared to a static equivalent monolithic beam. The lower global stiffness leads to higher deflections, which in turn leads to higher stresses for a given $G_{i n t}$.

However, since $\sigma_{\text {gross }}$ is inversely proportional to the squared beam height $h$ (see Eq. (1)), it is recommended to maximize the beam height in practice. 


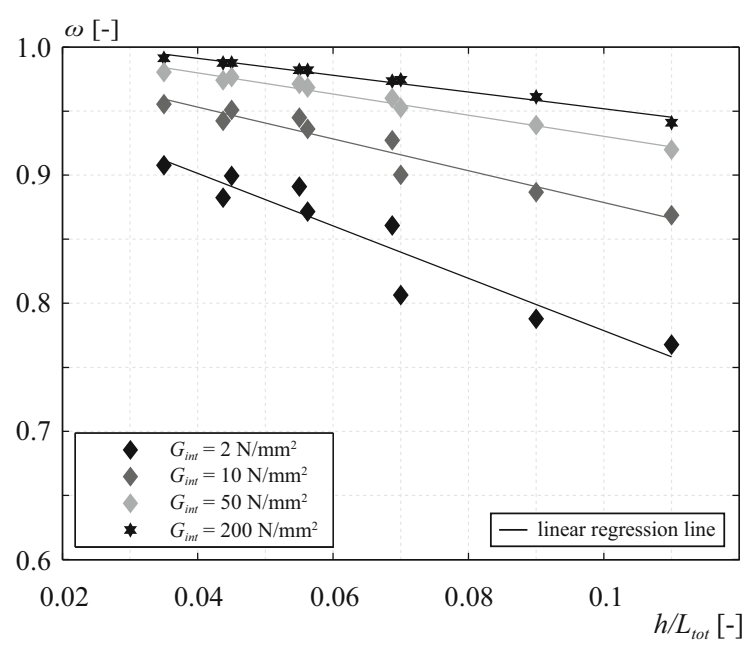

Fig. 10 Stiffness ratio $\omega$, based on the calculated global beam stiffness to the theoretical stiffness of a monolithic beam, variation with slenderness $h / L_{\text {tot }}$ for all conducted simulations of configurations L7_12_K2_...

(a)

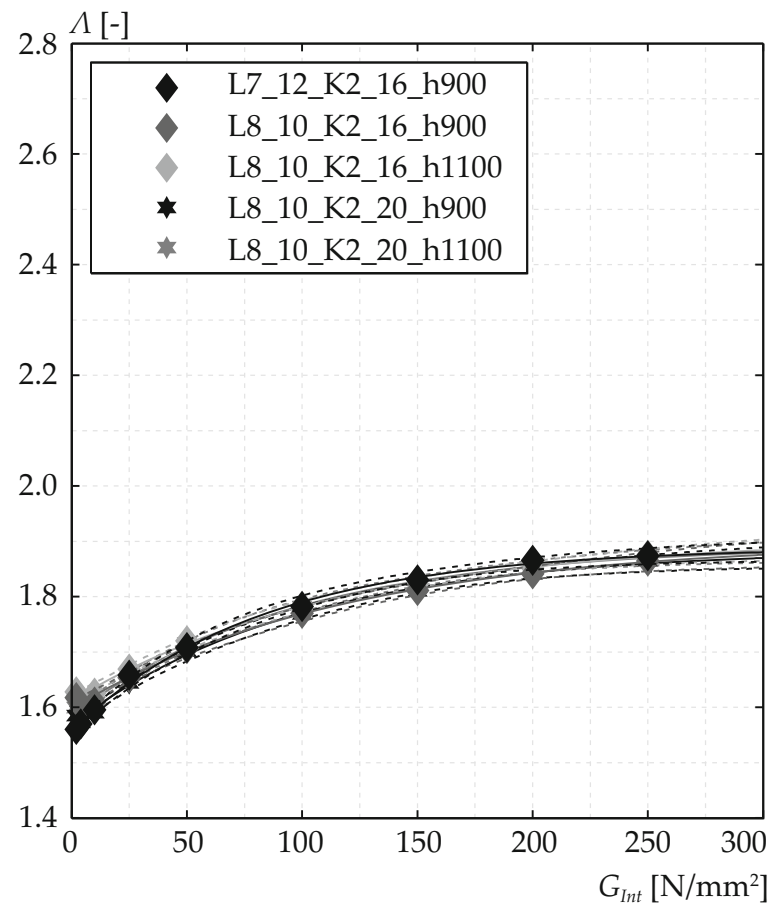

Fig. 11 Stress concentration factor $\Lambda$ variation with $G_{i n t}$ for the configurations L7_12_K2_16_h900, L8_10_K2_16_h900, L8_10_K2_16_h1100, L8_10_K2_20_h900 and L8_10_K2_
4.2 Eight- and nine-fold laminates with identical pane thicknesses

The simulations with eight- and nine-fold laminates with $10 \mathrm{~mm}$ thick panes show that in case of large $G_{\text {int }} \Lambda$ decreases from around 2.6-2.4 compared to the seven-fold laminate. The obtained $\Lambda$ for the different eight- and nine-fold laminates are illustrated in Figs. 11 and 12 , respectively.

This decrease is the result of two effects. Firstly, the panes are thinner and therefore the spliced pane carries less force, which has to be transferred in the region of a joint, leading to lower local effects and stress concentrations. Secondly, the spliced pane corresponds to a smaller portion of the total thickness of the beam, which leads to a lower eccentricity and therefore lower lateral bending. In principal, the same influences and ways as in case of conventional stress concentrations for geometric discontinuities can be applied. However, since spliced structural glass beams have an inhomo-

(b)

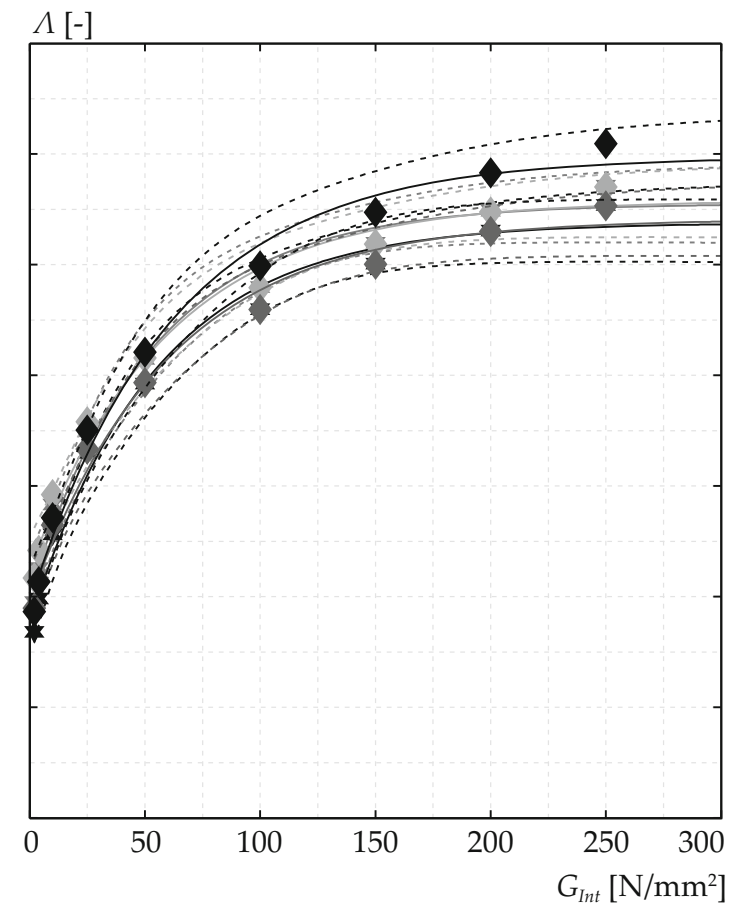

20_h1100 with the estimated nonlinear regression function: a inner spliced joint (S2 or S6/S7 spliced); b outer spliced joint 
(a)

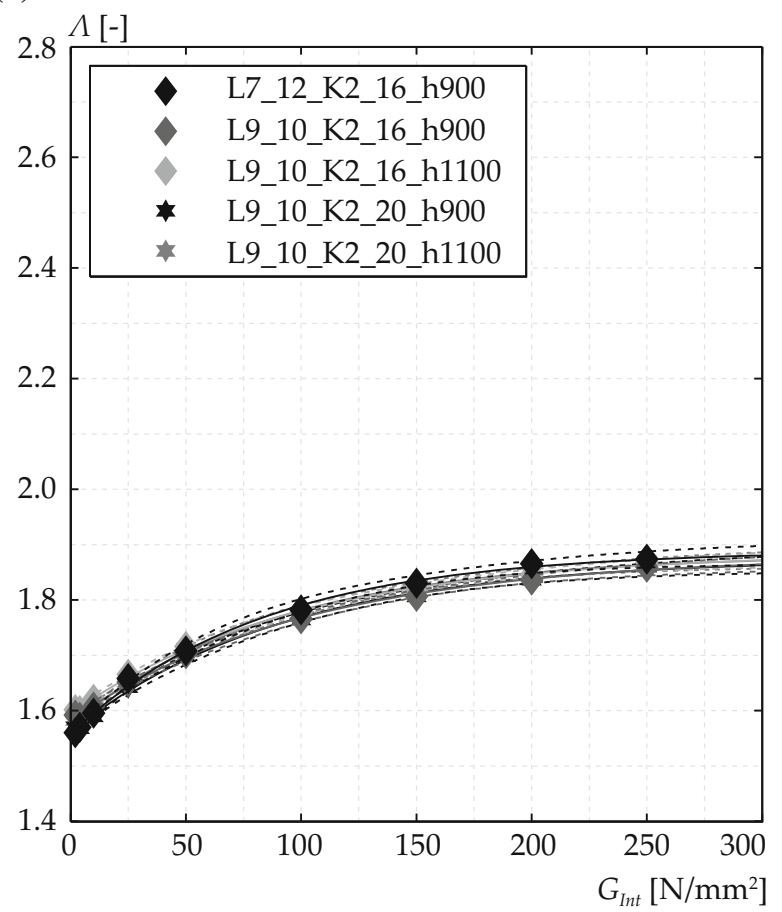

Fig. 12 Stress concentration factor $\Lambda$ variation with $G_{i n t}$ for the configurations L7_12_K2_16_h900, L9_10_K2_16_h900, L9_10_K2_16_h1100, L9_10_K2_20_h900 and L9_10_

geneous cross section, these influences have a different weighting, which depends on $G_{\text {int }}$.

Contrary to the advantage of eight- and nine-fold laminates over seven-fold laminates, there are also several drawbacks:

- The number of glass panes and interlayer material increases, leading to higher material and manufacturing costs.

- More joints are required and, consequently, more stress concentrations arise within the beam.

This leads to a trade-off when deciding how many glass layers should be used. This trade-off has to be resolved in each project on an individual basis.

In the following sections, two possibilities are introduced in an attempt to reduce the stress concentration factors and the aforementioned drawbacks for eightand nine-fold laminates.

\subsection{Optimized joints}

Two optimizing alternatives are presented here as an alternative to the eight- or nine-fold laminates. Firstly, (b)

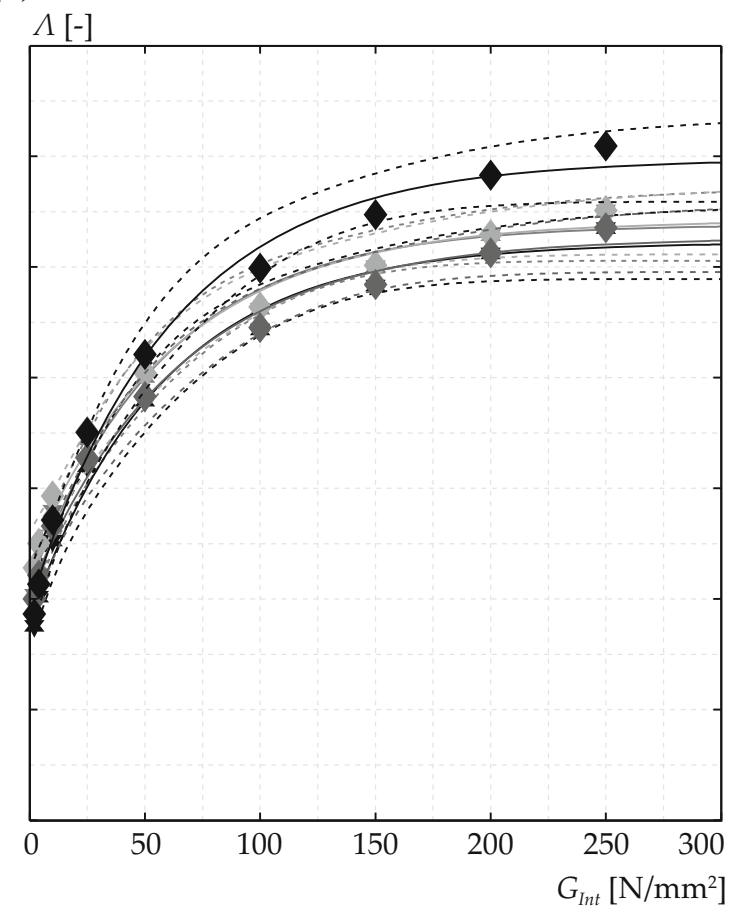

K2_20_h1100 with the estimated nonlinear regression function: a inner spliced joint (S2 or S6/S8 spliced); b outer spliced joint

the use of thinner outer panes is discussed and, secondly, the use of an intermediate pane is considered. The considered configurations are listed in Table 3.

\subsubsection{Thinner outer panes}

By using thinner outer panes (namely panes $\mathrm{S} 1$ and $\mathrm{S} 7$ in case of a seven-fold laminate) the force, which has to be transferred from the outer panes to the inner panes in outer spliced joints, is reduced. Hence, lower lateral bending action and lower local effects are expected, which will result in a decrease of $\Lambda$.

The FE simulations conducted with thinner outer panes are listed in the first half of Table 3.

The distribution of $\Lambda$ for the different configurations and $G_{i n t}$ are illustrated in Fig. 13a for inner spliced joints and Fig. 13b for outer spliced joints. In order to compare the results to the previous investigated configurations, configuration L7_12_K2_20_h900 are also included as reference configuration.

Inner spliced joints In the case of inner spliced joints, higher $\Lambda$ values are obtained as in the reference con- 
Table 3 Overview of FE simulations conducted with thinner outer panes and intermediate panes

\begin{tabular}{|c|c|c|c|c|c|c|c|c|c|c|c|c|}
\hline Name & $\begin{array}{l}n \\
(-)\end{array}$ & $\begin{array}{l}h \\
(\mathrm{~mm})\end{array}$ & $\begin{array}{l}t_{p} \\
(\mathrm{~mm})\end{array}$ & $\begin{array}{l}t_{i} \\
(\mathrm{~mm})\end{array}$ & $\begin{array}{l}b_{\text {stat }} \\
(\mathrm{mm})\end{array}$ & $\begin{array}{l}b_{t o t} \\
(\mathrm{~mm})\end{array}$ & $\begin{array}{l}L_{\text {inter }} \\
(\mathrm{mm})\end{array}$ & $\begin{array}{l}L_{J} \\
(\mathrm{~mm})\end{array}$ & $\begin{array}{l}L_{1} \\
(\mathrm{~mm})\end{array}$ & $\begin{array}{l}L_{t o t} \\
(\mathrm{kN})\end{array}$ & $F$ & $\begin{array}{l}h / L_{O} \\
(-)\end{array}$ \\
\hline L7_12_10_K2_20_h900 & 7 & 900 & $12 / 10$ & 1.52 & 80 & 89 & - & 1285.7 & 1000 & 20,000 & 324.0 & 0.35 \\
\hline L7_12_8_K2_20_h900 & 7 & 900 & $12 / 8$ & 1.52 & 76 & 85 & - & 1285.7 & 1000 & 20,000 & 307.8 & 0.35 \\
\hline L8_10_8_K2_20_h900 & 8 & 900 & $10 / 8$ & 1.52 & 76 & 87 & - & 1125.0 & 1000 & 20,000 & 307.8 & 0.27 \\
\hline L9_10_8_K2_20_h900 & 9 & 900 & $10 / 8$ & 1.52 & 86 & 98 & - & 1000.0 & 1000 & 20,000 & 348.3 & 0.30 \\
\hline L7_12_K2_20_h900_k50 & 7 & 900 & 12 & 1.52 & 84 & 93 & 50 & 1285.7 & 1000 & 20,000 & 340.2 & 0.35 \\
\hline L7_12_10_K2_20_h900_k50 & 7 & 900 & $12 / 10$ & 1.52 & 80 & 89 & 50 & 1285.7 & 1000 & 20,000 & 324.0 & 0.35 \\
\hline L7_12_10_K2_20_h900_k75 & 7 & 900 & $12 / 10$ & 1.52 & 80 & 89 & 75 & 1285.7 & 1000 & 20,000 & 324.0 & 0.35 \\
\hline L9_10_8_K2_20_h900_k50 & 9 & 900 & $10 / 8$ & 1.52 & 86 & 98 & 50 & 1000.0 & 1000 & 20000 & 348.3 & 0.35 \\
\hline
\end{tabular}

(a)

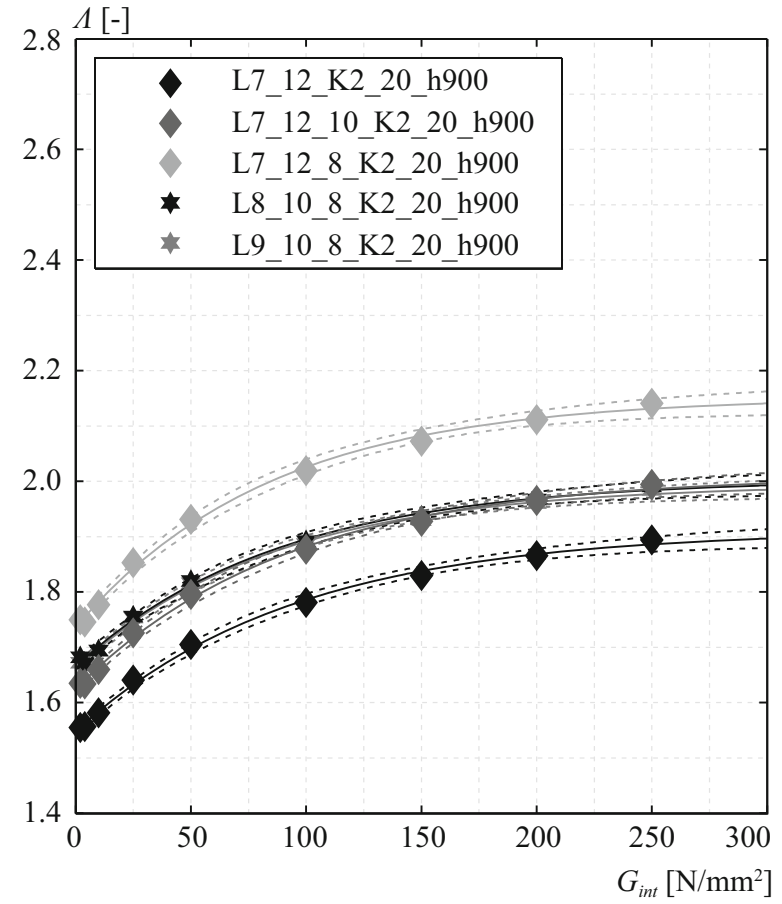

Fig. 13 Stress concentration factor $\Lambda$ variation with $G_{\text {int }}$ for the configurations L7_12_K2_20_h900, L7_12_10_K2_20_h900, L7_12_8_K2_20_h900, L8_10_8_K2_20_h900 and L9_10_

figuration. This increase can be explained on the one hand side with higher stresses from the local effects, which can be approximated as a local lateral bending (see Ringli and Vogel 2016) where the thickness goes by the power of two into the calculation of the stresses. On the other hand, the force that is transferred from the thicker spliced pane leads to higher stresses in the outer pane due to the reduced cross section in comparison to the reference configuration. (b)

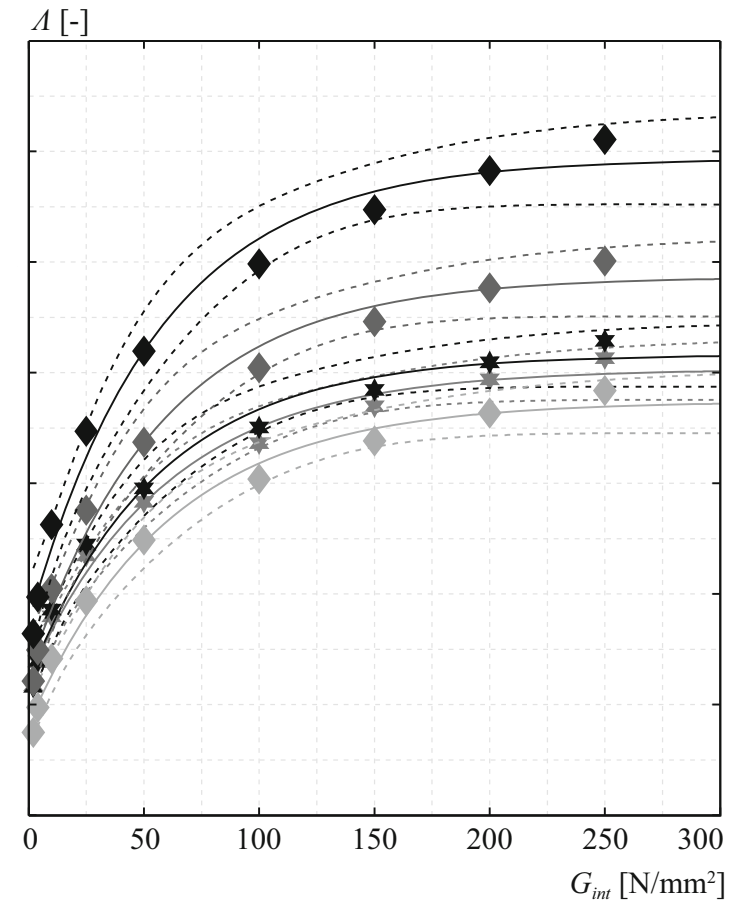

8_K2_20_h900 with the estimated nonlinear regression function: a inner spliced joint (second or second last pane spliced); b outer spliced joint

Outer spliced joints In the case of outer spliced joints, $\Lambda$ decreases because less force is transferred from the spliced pane to the continuous panes, leading to a lower lateral bending and a lower local bending. Consequently, lower $\sigma_{\max }$ are obtained and $\Lambda$ reduces in relation to the reference configuration.

In summary, thinner outer panes lead to lower $\Lambda$ in the case of outer spliced joints and to higher $\Lambda$ in the case of inner spliced joints, which can be higher than in 
Fig. 14 Concept of the intermediate pane (a)

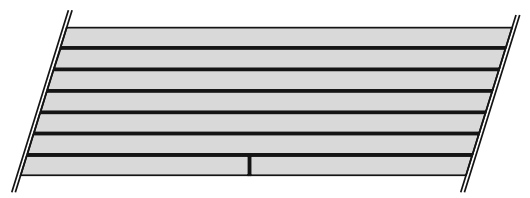

(b)

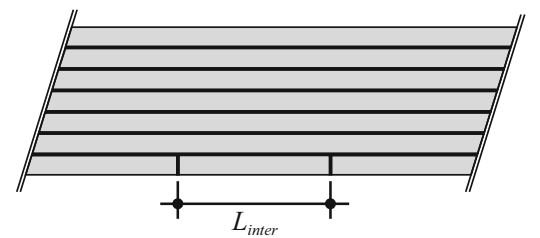

the case of outer spliced joints. Hence, configurations are possible where $\sigma_{\max }$ occurs in the cross section of inner spliced joints and not in the cross section of outer spliced joints. As a consequence, inner spliced joints have to be analyzed in detail as well and a blind focus on improving the stress distribution of outer spliced joints is not advisable.

\subsubsection{Intermediate pane and thinner outer panes}

The motivation to use an intermediate pane in outer spliced joints is to ensure that the transferred force spreads more equally within the adjacent pane. In conventional close fitted joints, the force is transferred before and retransferred right after the joint. This leads to higher stresses at the edge of the adjacent pane, because the force has no motivation to spread equally over the pane thickness, since it is retransferred right behind and therefore sticks to the edge in order to minimize its path through the adjacent pane. This sticking to the edge leads to a large local bending action since the distance of the force to the axis of the adjacent pane increases.

The concept of intermediate panes is schematically illustrated in Fig. 14.

The intermediate panes investigated had a length of $L_{\text {inter }}=50$ and $75 \mathrm{~mm}$, respectively. The FE simulations conducted considering intermediate panes are listed in the second half of Table 3.

The obtained $\Lambda$ values are illustrated in Fig. 15 for the different configurations.

Inner spliced joint In the case of an inner spliced joint, the obtained $\Lambda$ does not differ significantly from the reference configuration. This was expected, since no changes were made to the inner spliced joint.

Outer spliced joint In the case of an outer spliced joint, the stress concentrations could be reduced significantly. As explained in the motivation to use intermediate panes, these lead to a smoother flow of forces, which results in lower stress concentrations.
The combination of intermediate panes with thinner outer panes results in a further reduction of the stress concentrations in outer spliced joints. However, this reduction is such significant that $\Lambda$ drops under the value for inner spliced joints. Hence, the local improvement of the outer spliced joint is not a real improvement considering the entire beam.

\subsection{Application and comparison}

Different configurations with $L_{t o t}=20 \mathrm{~m}$ and $h=$ $900 \mathrm{~mm}$ are compared in this section, assuming different load scenarios with their related $G_{\text {int }}$.

Due to the characteristic material behavior of SG, different load scenarios have to be considered in the design process. In this study, the following three load scenarios were considered:

Load scenario 1: Placement of the beam during winter (temperature of the beam $15{ }^{\circ} \mathrm{C}$ ) and heavy snow fall after one month.

- $G_{\text {int }, E G, A}=100 \mathrm{~N} / \mathrm{mm}^{2}$ (load duration: 1 month)

- $G_{\text {int,snow }}=150 \mathrm{~N} / \mathrm{mm}^{2}$ (load duration: 1 day)

Load scenario 2: The beam is 10 years old on a hot summer day (temperature of the beam $50{ }^{\circ} \mathrm{C}$ ).

- $G_{\text {int }, E G, A}=2 \mathrm{~N} / \mathrm{mm}^{2}$ (load duration: 10 years)

Load scenario 3: Assumptions from Trösch (2015) and DIBt (2011).

- $G_{i n t, E G, A}=2 \mathrm{~N} / \mathrm{mm}^{2}$

- $G_{\text {int,snow }}=60 \mathrm{~N} / \mathrm{mm}^{2}$

The following stresses at the lower edge are assumed resulting from the loads (based on L7_12_K2_20_ h900):

- Due to own weight: $10 \mathrm{~N} / \mathrm{mm}^{2}$

- Due to additional dead load: $12 \mathrm{~N} / \mathrm{mm}^{2}$

- Due to snow: $15 \mathrm{~N} / \mathrm{mm}^{2}$ 
(a)

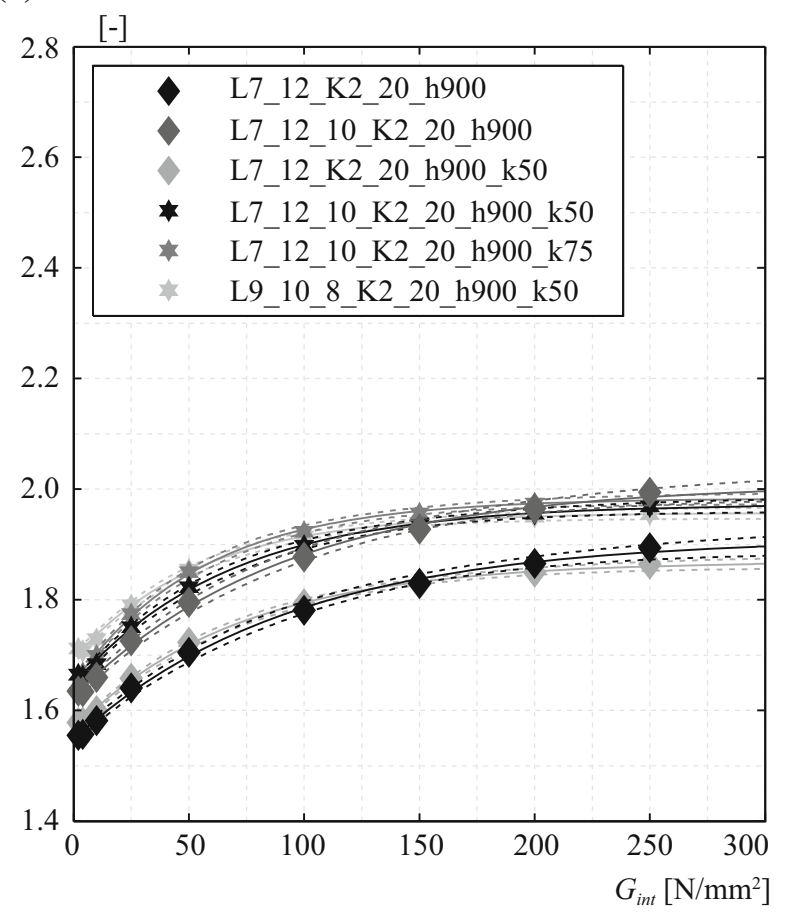

Fig. 15 Stress concentration factor $\Lambda$ variation with $G_{\text {int }}$ for the configurations L7_12_K2_20_h900, L7_12_10_K2_20_h900, L7_12_K2_20_h900_k50, L7_12_10_K2_20_h900_k50， L7

These assumptions lead to $\sigma_{\text {gross, tot }}=37 \mathrm{~N} / \mathrm{mm}^{2}$ for load scenarios 1 and 3 and $\sigma_{\text {gross, tot }}=22 \mathrm{~N} / \mathrm{mm}^{2}$ for load scenario 2.

For the other configurations with more glass layers or thinner outer panes, the previously assumed stresses were adjusted, since a higher $b_{\text {stat }}$ leads to lower stresses given additional dead load and snow load.

The different $\sigma_{\max }$ are calculated for each configuration and the three different load scenarios using Eqs. (3) and (4) and Figs. 8, 9, 13 and 15. The obtained $\sigma_{\max }$ are illustrated in Fig. 16.

Figure 16 shows that $\sigma_{\max }$ can be reduced significantly for a given load scenario by carefully designing the configuration. In addition, it is shown that the stresses vary widely between the load scenarios. Hence, it is crucial to define the $G_{\text {int }}$ within the correct range in order to result in a safe design.

In some cases, the line representing the inner spliced joints lies above the line representing the outer spliced joint. In such cases, the location of the critical stress concentration moves from an outer spliced joint to an (b)

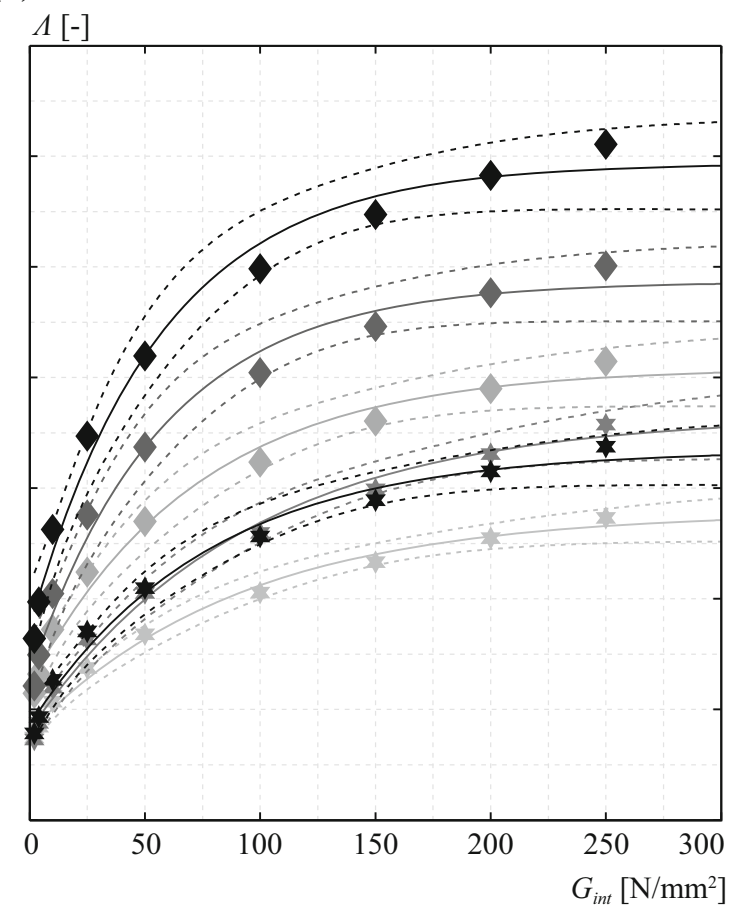

_12_10_K2_20_h900_k75 and L9_10_8_K2_20_h900_k50 with the estimated nonlinear regression function: $\mathbf{a}$ inner spliced joint (second or second last pane spliced); b outer spliced joint

inner spliced joint. As a consequence, both inner and outer spliced joints have to be analyzed in detail.

Furthermore, Fig. 16 shows that the effectiveness of the joint optimization depends on the chosen scenario. In load scenarios 1 and 3, the stresses and strains could be reduced significantly. In load scenario 2 , however, only marginal changes were observed in the obtained stresses.

\section{Summary and conclusion}

This investigation shows that $\sigma_{\max }$ highly depends on $G_{\text {int }}$. This dependence is nonlinear and sensitive to cases with low $G_{i n t}$. Hence, the interlayer stiffness, which is the basis for the design, has to be chosen very carefully in dependence on the particular project considering the specific environmental conditions. It is strongly recommended to analyze at least two different load scenarios during the design process: one with a combination of high and one with a combination of low interlayer stiffness. 
Fig. 16 Maximal tensile stress for different configurations and load scenarios

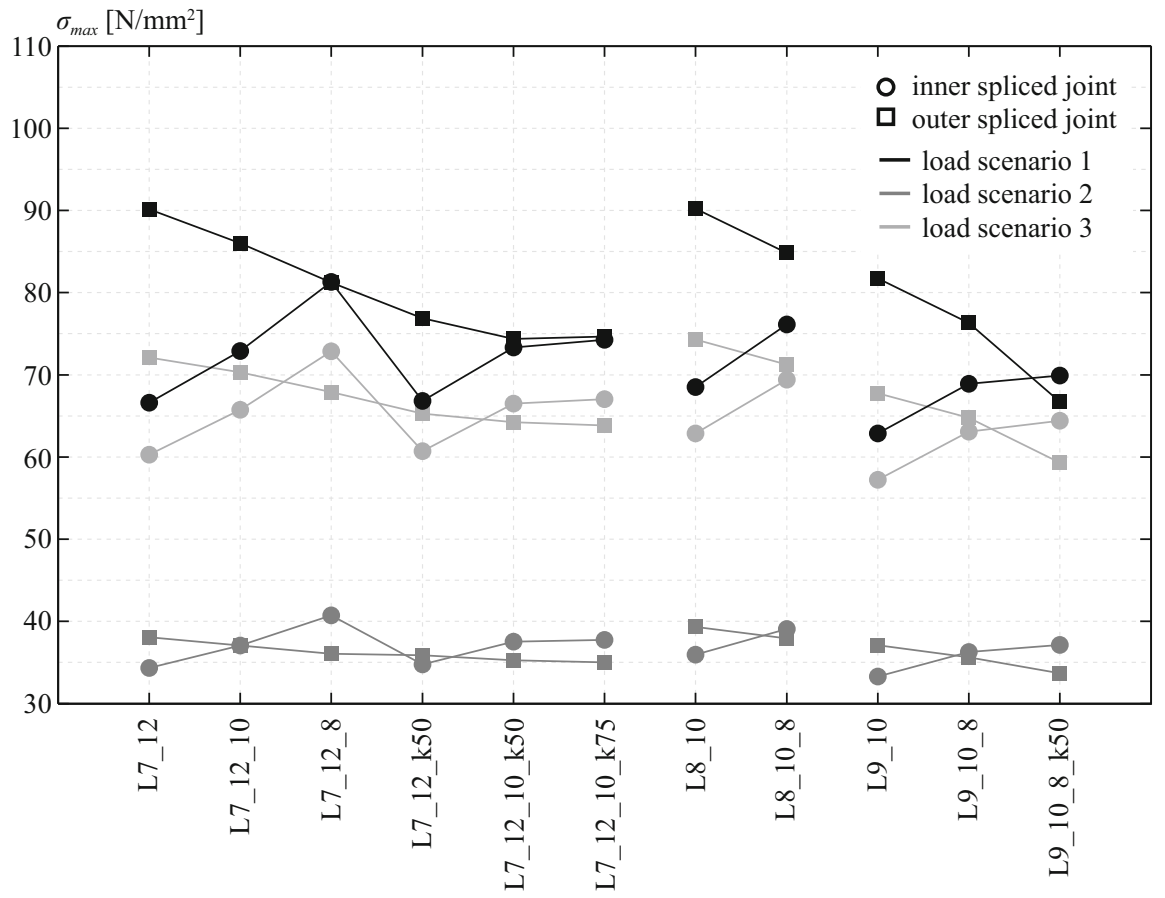

The numerical simulations show that the stress concentrations assuming low interlayer stiffness are significant lower than for high interlayer stiffness. Hence, would it be a good idea to use PVB as interlayer material instead of SG, since PVB has a significant lower stiffness compared to SG (see e.g. Kuntsche et al. (2015) or Trösch (2015)) to reduce the stress concentrations? The answer is no because a minimal shear coupling between the individual panes is needed for spliced laminated glass beams in order to keep the panes in place and to transfer force between neighboring panes.

In general, the local load-bearing behavior in the region of an outer spliced joint can be approximated by assuming the lower edge of the beam as a region of pure tensile action and analyzing that area according to the analytical model introduced in Ringli and Vogel (2016). However, the analytical model is not able to consider interaction between neighboring joints and, therefore, the results may differ from the results obtained by FE simulations for cases with low interlayer stiffness. Due to this restriction, the analytical model cannot be used to calculate the actual stresses and strains, but is useful in order to validate the results obtained from FE simulations. In addition, it is suited to obtain a better understanding of the different influences and effects that describe the load-bearing behavior of such struc- tures. This enables the design engineer to develop optimized structures and especially optimized joint details.

In this paper, the following two joint optimizations were introduced, which are suited to lower $\Lambda$ to some extent:

- The predicted $\sigma_{\max }$ in case of outer spliced joints can be reduced by using thinner outer panes. Two effects lead to this reduction: Firstly, less force has to be transferred from the spliced pane to the continuous panes, such that the influence of the local effects is reduced. Secondly, the eccentricity of the residual force is reduced, which leads to a lower lateral bending action. However, thinner outer panes have the following drawback: In the case of inner spliced joints, thinner outer panes increase $\Lambda$ due to the local effects. Hence, it is recommended to use just one step thinner outer panes compared to the inner panes. In addition, it is recommended to not only focus on outer spliced joints, but also to consider inner spliced joints, especially in cases with low interlayer stiffness.

- Intermediate panes dividing an outer spliced joint into two joints also result in a reduction of $\Lambda$. The intermediate pane allows a more uniform transfer 
of force from the spliced pane to the continuous pane before retransferring to the spliced pane. This behavior results in a more steady force path, which leads to a lower $\Lambda$.

The results presented here might also be used to gain a better understanding of the post-failure behavior of non-spliced laminated glass beams. In a first stage, damaged non-spliced laminated glass beams could be approximated by spliced glass beams where the cracked pane corresponds to a spliced pane. This assumptions may be legitimate if annealed glass is used, since then the crack is limited to small area. In case of a damaged fully tempered glass, however, this assumption is not advisable because the cracks in the damaged pane are distributed in a large area (see e.g. Trösch 2015) and therefore, the entire damaged pane eludes force.

\section{References}

Belis, J., Depauw, J., Callewaert, D., Delincé, D., Van Impe, R.: Failure mechanisms and residual capacity of annealed glass SPG laminated beams at room temperature. Eng. Fail. Anal. 16, 1866-1875 (2009)

Bischoff, G.: Verbundglas. European patent specification, 0575 957 B1 (1993)

Boltzmann, L.: Zur Theorie der elastischen Nachwirkung. Wien. Ber., reprint, Wissenschaftliche Abhandlungen von Ludwig Boltzmann, Band 1. Chelsea Publishing Company, New York (1874)

Callewaert, D.: Stiffness of Glass/Ionomer laminates in structural applications. $\mathrm{PhD}$ thesis, Ghent University (2012)

DIBt: Allgemeine bauaufsichtliche Zulassung Zulassungsgegenstand: Verbund-Sicherheitsglas aus Sentry Glas SGP 5000 mit Schubverbund. German Center of Competence for Construction (DIBt) (2011)
Hess, R.: Glasträger. Technical report, ETH Zurich (2000)

Kuntsche, J., Schuster, M., Schneider, J., Langer, S.: Viscoelastic properties of laminated glass interlayers: theory and experiments. Proc. Glass Perform. Days Finl. 2015, 143-147 (2015)

Kuraray: SentryGlas ${ }^{\circledR}$ Ionoplast Interlayer Specifying and Technical Data (2014)

Meissner, M., Bucak, Ö.: Trag- und Resttragfähigkeitsuntersuchungen an Verbundglas mit der Zwischenlage "SentryGlas Plus". Technical report, University of Applied Science Munich (2005)

Pilkey, W.D., Pilkey, D.F.: Peterson's Stress Concentration Factors, 3rd edn. Wiley, New York (2008)

Puller, K.: Untersuchung des Tragverhaltens von in die Zwischenschicht von Verbundglas integrierten Lasteinleitungselementen. PhD thesis, University of Stuttgart (2012)

Ringli, R., Vogel, T.: An analytical model to estimate the maximum tensile stress in spliced laminates under tensile action-using the example of a fivefold laminate. In: Bulletin of the University Politehnica of Bucharest, Series D Mechanical Engineering, accepted for publication (2016)

Ringli, R., Vogel, T.: Load-bearing behavior of spliced beams under tensile load, Glass Performance Days, Tampere, Finland, 368-372 (2015)

Schuler, C.: Einfluss des Materialverhaltens von Polyvinylbutyral auf das Tragverhalten von Verbundsicherheitsglas in Abhängigkeit von Temperatur und Belastung. PhD thesis, Technical University of Munich (2003)

Seber, G.A.F., Wild, C.J.: Nonlinear Regression. Wiley, Hoboken (2003)

Trösch, E.: Tragverhalten von überlappend laminierten Verbundglasträgern für grosse Spannweiten. PhD thesis, ETH Zurich (2015)

Wellershoff, F.: Bemessungsschubmodulwerte für Verbundglasscheiben. Stahlbeton 76, 177-188 (2007) 